\title{
Screening the Hub Genes and Analyzing the Mechanisms in Discharged COVID-19 Patients Retesting Positive through Bioinformatics Analysis
}

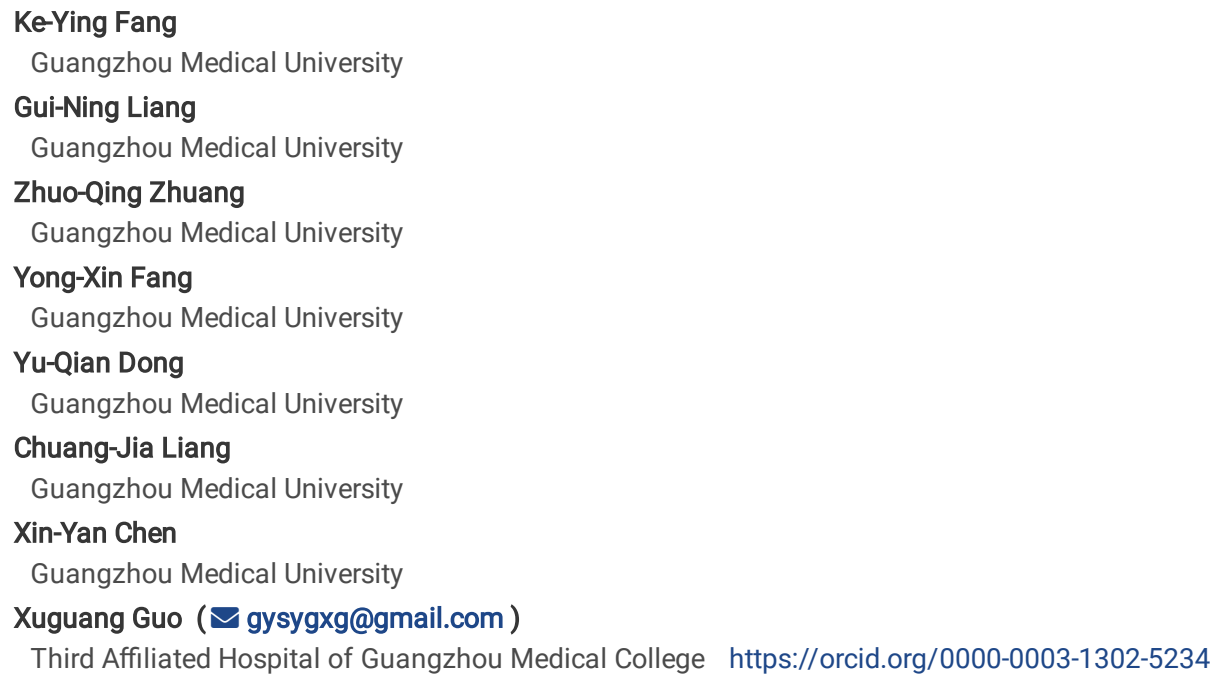




\section{Abstract}

Background: With the worldwide spread of COVID-19, people's health and social order have been exposed to enormous risks. After encountering patients who test positive again after discharge, our study analyzed the pathogenesis to further assess the risk and possibility of virus reactivation.

Methods: A separate microarray was acquired from the Integrated Gene Expression System (GEO), and its samples were divided into two groups: a "convalescent-RTP" group consisting of recovery and "retesting-positive" (RTP) patients (group CR) and a "health-RTP" group consisting of healthy control and RTP patients (group HR). The enrichment analysis was performed with R software, obtaining the gene ontology (GO) and Kyoto pluripotent stem cells (KEGG) of the genes and genomes. Subsequently, the protein-protein interaction (PPI) networks of each group were established and the hub genes were discovered using the cytoHubba plug-in.

Results: In this study, 20 differentially expressed genes were identified, and 6622 genes were identified in the group CR, consisting of 5003 up-regulated and 1619 down-regulated genes. Meanwhile, 7335 genes were screened in the group HR, including 4323 up-regulated and 3012 down-regulated ones. The GO and KEGG analysis of the two groups revealed significant enrichment of these differentially expressed genes in pathways associated with immune response and apoptosis. In the PPI network constructed, 10 hub genes in group CR were identified, including TP53BP1, SNRPD1, SNRPD2, SF3B1, SNRNP200, MRPS16, MRPS9, CALM1, PPP2R1A, YWHAZ. Similarly, TP53BP1, RPS15, EFTUD2, MRPL16, MRPL17, MRPS14, RPL35A, MRPL32, MRPS6, POLR2G were selected as hub genes.

Conclusions: Using the messenger ribonucleic acid (mRNA) expression data from GSE166253, we explore the pathogenesis of retesting positive in COVID-19 from the immune mechanism and molecular level. We found TP53BP1, SNRPD1 and SNRPD2 as hub genes in RTP patients. Hence, their regulatory pathway is vital to the management and prognostic prediction of RTP patients, rendering the further study of these hub genes necessary.

\section{Introduction}

The coronavirus disease 2019 (COVID-19), induced by the novel coronavirus, severe acute respiratory syndrome coronavirus 2 (SARS-CoV-2), has emerged as a global public health crisis. Typical symptoms of the disease are fever, sore throat, fatigue, cough or dyspnea ${ }^{[1]}$; droplet transmission is the main route of transmission, with close contact providing an opportunity for such virus transfer ${ }^{[1,2]}$. As of October 1,2021 , there are $233,136,147$ confirmed cases throughout the world, including 4,771,408 deaths reported by the World Health Organization $(\mathrm{WHO})^{[3]}$.

So far, a large number of patients have been cured and discharged from hospitals. However, some, described as "retesting-positive" (RTP) patients, were retested positive for SARS-CoV-2 after discharge. According to a systematic review, the RTP cases accounted for about $12 \%$ of discharged patients ${ }^{[4]}$ with the median duration of viral shedding in RTP patients after admission being $14.0^{[5]}$. Most of these patients' clinical results revealed moderate severity conditions along with clinical symptoms including cough, sputum production, headache and dizziness ${ }^{[6]}$. As for the cause of RTP, the related researches focus on the false-negative RT-PCR testing at discharge ${ }^{[5]}$ or the persistence of the virus and periodic shedding in the gastrointestinal tract ${ }^{[7]}$. However, there still remain concerns that discharged patients may be at risk of viral reactivation and should be considered potential sources of SARS-CoV-2 infection, which may become a public health crisis. Therefore, further exploration of the impaired immune function and pathogenesis is required.

Based on the previous study in immunology, a higher level of white blood count (WBC) was independently relevant to RTP by RT-PCR ${ }^{[8]}$. Besides, patients infected with COVID-19 also exhibited higher leukocyte numbers and elevated plasma pro-inflammatory cytokine levels ${ }^{[2]}$. Additionally, compared with convalescent patients, they presented lower levels in lactate dehydrogenase (LDH) at the first week after admission but higher levels in eosinophil count ${ }^{[9]}$. According to Yao MQ et al., patients presenting with decreased leukocyte, platelet and CD4+ T counts were at a higher risk of RTP ${ }^{[6]}$. Xiangying Ren et al. revealed that ribonucleic acid (RNA) of viral could still be detected in patients with SARS-CoV-2 specific immunoglobulin M (IgM) and immunoglobulin G (IgG) present[4]. Besides, a systematic review presented that it's predictive to recognize RTP through a combination of lower high-sensitivity C-reactive protein (hs$\mathrm{CRP}$ ) and higher WBC[8]. Overall, little is known about RTP in immunology and pathogenesis, and many results are in conflict with the previous study.

Therefore, we believe that it is necessary to analyze the biological information of the samples. The research results may further expand the insight into RTP events in COVID-19 by analyzing the RTP immunological characteristics and molecular pathogenesis genes of peripheral blood mononuclear cells containing RTP patients, convalescent patients and healthy control subjects.

\section{Materials And Methods}

\section{Data collection}

Aiming at analyzing the biological information of RTP patients, initially, we performed a search of the Gene Expression System (GEO) data using SARS-CoV-2 as the keyword (ncbi.nlm.nih.gov/geo/). Dataset GSE166253, contributed by Wang D et al., was found and used in our study(Table 1). The sequencing platform employed was the GLP20795 HiSeq X Ten (Homo sapiens). The dataset consisted of 10 retesting-positive (RTP) samples, six convalescent and 10 healthy ones, with the sample source containing the peripheral blood mononuclear cells (PBMCs) of human beings. To analyze the pathogenesis of RTP occurrence in COVID-19, we divided the samples into two groups: a "convalescent-RTP" group comprising of convalescent patients and RTP patients (group $\mathrm{CR}$ ) and a "healthy-RTP" group of healthy controls and RTP patients (group HR).

\section{Screening of DEGs}


After acquiring the gene expression data, the R pack (R Foundation for statistic computing) was utilized, and the Fold change (FC) and P-value were calculated to identify the differentially expressed genes (DEGs) from the two groups (CR and HR). Following this, in the $\mathrm{R}$ language program, $\mathrm{p}<0.05$ and $\mid$ log $2 \mathrm{FCl} \geq 1$ were designed as the threshold, and the genes that met the criteria were sifted out as DEGs. Finally, we constructed volcano plots and heatmaps to visualize all the DEGs of GSE166253.

\section{Functional Enrichment Analysis}

The GO annotation can be divided into three categories: biological process (BP), cellular component (CC) and molecular function (MF). In our study, we execute the GO function and KEGG pathway enrichment analysis for the DEGs, with the P-value $<0.05$ deemed statistically significant.

\section{Establishment of PPI and Screening of Hub Genes}

Exploring functional interactions between proteins is crucial to understanding the molecular mechanisms of RTP for the COVID-19 infection. The Search Tool for the Retrieval of Interacting Genes (STRING), an online tool to assess and integrate PPI information, contains physical and functional associations ${ }^{[10]}$. To find the potential correlations to the DEGs, we employed STRING to map their PPI network. If the interaction score was $>0.4$, then it will permit protein interaction. To draw an interaction of DEGs, the Cytoscape software was used to visualize the PPI network. To examine the protein interaction further, we employed this on the basis of the PPI associations to screen out hub genes. Finally, the immune infiltration analysis was performed for the two groups to further confirm the results of our study. The results of the immunoblotting analysis are attached to the supplementary document.

\section{Results}

\section{Screening of DEGs}

Downloading the GSE166253 dataset from the GEO database, we conducted differential expression analysis using the R language. In group CR, 20376 DEGs were identified, containing 5003 up-regulated and 1619 down-regulated genes (Table 2). Simultaneously, 20376 DEGs were found in group HR, consisting of 4323 up-regulated and 3012 down-regulated genes (Table 3). Subsequently, volcano plots and heatmaps were performed to present the DEGs in both groups (Fig.1 and Fig.2). The volcano plots depicted upregulated (blue) and downregulated (red) genes, while the heatmaps presented the expression levels of the DEGs.

\section{Functional Enrichment}

With regard to the CR group (Table 4), the GO analysis in the biological processes demonstrated that the DEGs were significantly enriched in the processes that neutrophils are involved in, such as the activation of neutrophil, neutrophil-mediated immune and neutrophil activation participated in immune response. As for the cellular components, the mitochondrial inner membrane and mitochondrial protein complex were predominantly recognized. Besides, the terms of the most enriched in molecular functions were associated with transcription coactivator activity as well as magnesium ion binding (Fig.3a). Moreover, according to the KEGG enrichment analysis results of group CR (Table 5), three significant pathways of DEGs were identified, containing endocytosis, nucleotide-binding oligomerization domain-like receptors (NOD-like receptor) signal path and tumor necrosis factor (TNF) signal path (Fig.3b).

Similarly, the results in group HR were shown as follows (Table 6): the GO enrichment in biological processes revealed that DEGs were primarily located on neutrophil activation, neutrophil-mediated immunity as well as neutrophil activation participating in immune response. In terms of the category of cellular components, the mitochondrial inner membrane, mitochondrial matrix and nuclear speck were chiefly enriched. For molecular functions, the mitochondrial inner membrane, mitochondrial matrix and nuclear speck were significantly identified (Fig.3c). In the KEGG pathway enrichment analysis (Table 7), a total of 10 key pathways were found, especially the pathways associated with prion disease, Huntington's disease and Parkinson's disease, which take part in the corresponding neurodegenerative disorder (Fig.3d).

\section{Establishment of PPI and Screening of Hub Genes}

We constructed the PPI networks for both the CR and HR groups, after which the top 10 genes with the highest degree of connectivity were determined using the Cytoscape and cytoHubba plugin (Fig.4 and Fig.5). In group CR, 1246 nodes and 5665 edges were included in the PPI network for up-regulated DEGs (Fig.4a), while 1060 nodes and 613 edges were found in the PPI network of down-regulated DEGs (Fig.4b). Afterwards, TP53BP1, SNRPD1, SNRPD2, SF3B1, SNRNP200, MRPS16, MRPS9, CALM1, PPP2R1A, YWHAZ were identified as hub genes (Fig.4C). As for group HR, 1223 nodes and 1257 edges were found in the PPI network of up-regulated DEGs (Fig.5a), whereas for down-regulated DEGS, 732 nodes and 228 edges were found in the PPI network (Fig.5b). With the same methods, 10 hub genes, including TP53BP1, RPS15, EFTUD2, MRPL16, MRPL17, MRPS14, RPL35A, MRPL32, MRPS6, POLR2G, were screened out and their interactions were displayed in Fig.5c.

\section{Discussion}

With the spread of the COVID-19 epidemic, the situation of RTP for SARS-CoV-2 after discharge renders the management of discharged patients a difficult problem ${ }^{[11]}$, potentially leading to a public health crisis. At present, there is still no definitive conclusion about the cause of RTP, and the possibility of their reinfection and disease transmission cannot be ruled out. In this study, the peripheral blood samples of RTP patients, convalescent patients and healthy people were divided into two groups-CR and HR groups, and the immune mechanism of COVID-19 RTP was explored based on the results of the enrichment analysis. 
Through the $\mathrm{GO}$ analysis for both the groups, the immune processes' neutrophils involved and the mitochondrial function and contents were significantly enriched. As Cynthia Magro et al. studied, patients with COVID-19 had markedly increased neutrophils ${ }^{[12]}$. The extracellular bactericidal networks (NETs) of SARS-CoV-2-activated neutrophils are considered important in the progress of the COVID-19 infection, and releasing NETs in lung tissue could result in the death of lung epithelial cells ${ }^{[13]}$, indicating that the immune processes that neutrophils are involved in RTP patients may be more activated in lysing infected cells and release the virus. The mitochondria plays a variety of interrelated functions in cellular metabolism, producing adenosine triphosphate (ATP) and many biosynthesis intermediates, as well as participating in stress responses such as autophagy and apoptosis ${ }^{[14]}$. Studies have pointed out that the monocytes of patients with COVID-19 accumulate dysfunctional mitochondria, resulting in their metabolic defects, decreased OXPHOS and glycolysis, impaired oxidative burst, altered immune response and possibly increased susceptibility to secondary infections ${ }^{[15]}$. RTP patients may experience more intense mitochondrial dysfunction. Additionally, mitochondrial dysfunction was a key factor in neurodegenerative diseases ${ }^{[14]}$, which also corresponded to the KEGG-rich pathway in the HR group (prion disease, Huntington's disease)-this may be related to the prognosis of RP patients.

Apart from neurodegenerative diseases, the KEGG analysis of both the CR and HR groups also revealed that endocytosis, the NOD-like receptors and TNF pathways were the main enrichment pathways. Endocytosis plays a vital role in the infection of disease and restoration of health. Recent studies have suggested that the key event supporting the SARS-CoV-2 infection may be the endocytosis of viral particles ${ }^{[16]}$, which may be more intense in RTP patients. NOD-like receptors (NLRs), a class of pattern recognition receptors (PRRs) ${ }^{[17]}$, can recognize ligands from microbial pathogens, host cells and environmental sources, then activate an inflammatory response ${ }^{[18]}$. Similar to mitochondria, NLRs play a significant part in regulating apoptosis, acting as a key regulator of apoptosis as well as aiding in early development ${ }^{[16]}$ and directing autophagy by recruiting ATG16L1 to the plasma membrane at the site of the bacterial entry sites $^{[19]}$. The TNF superfamily is a multifunctional proinflammatory cytokine that participates in the processes of cell survival, apoptosis induction,

inflammatory response and cell differentiation ${ }^{[20]}$. In the SARS-CoV-2 infection, innate immune cells will produce a variety of inflammatory cytokines, but only the combination of TNF-a and IFN-y can damage vital organs by inducing the death of inflammatory cells ${ }^{[21]}$. In summation, the NOD-like receptors and TNF pathways may contribute extensively to RTP patients through apoptosis and inflammatory response.

Since the results of the HR group cannot exclude the interference with the COVID-19 infection, we selected the hub genes of the CR group for discussion next, with the HR group taken as verification. TP53BP1 was enriched in both groups, and its expression in the CR group was downregulated. TP53BP1, one of the regulator genes of TP53, is known to be highly associated with the DNA damage signal pathway, checkpoint signal and DNA repair capacity ${ }^{\text {[2] }}$. Moreover, it has been associated with cancer and neurological diseases ${ }^{[22,23]}$. In the immune system, DNA repair factors TP53 can support immunoglobulin class switch recombination ${ }^{[24]}$. B cells are critical for the production of antibodies and protective immunity to viruses, and Tan et al. discovered a stronger antibody response is related to delayed viral clearance as well as disease severity ${ }^{[25]}$. According to their research, the downregulation of TP53BP1 resulted in a lower antibody response, indicating a higher level of viral clearance in RTP patients. In a study on the mechanisms of the maintenance of foot-and-mouth disease (FMD) virus persistence, TP53BP1 in the persistently positive group was also significantly downregulated. It was reported that the downregulation of TP53BP1 could inhibit the Th17 response, natural killer cell (NK cell) cytotoxicity and apoptosis ${ }^{[26]}$. The main effector of Th17 cells is IL-17, while the lymphocytes in COVID-19 patients can produce more IL-17, and blocking IL-17 could be a novel therapeutic strategy for COVID-19 ${ }^{\text {[27] }}$. The lower expression of IL-17 in RTP patients may lead to milder symptoms. Preliminary studies of patients with severe COVID-19 indicate that the decrease in the number or function of NK cells will reduce the clearance of infected and activated cells ${ }^{[28]}$. Moreover, the limitation of apoptosis and reduction in clearing infected and activated cells may lead to the slower death of infected cells, resulting in the longer discharge of RNA of SARS-CoV-2 in RTP patients.

SNRPD1 and SNRPD2 are both up-regulated in group CR, with the main regulatory pathway of the former being the mRNA splicing ${ }^{[29]}$. The study confirmed that spliceosome machinery is a replicative machinery of SARS-CoV-2 for evading host challenges, which may prove that the replication activity of SARS-CoV2 is more active in RTP patients ${ }^{[30]}$. According to Xiaofeng Dai et al., SNRPD1 over-expression is observably connected to genes involved in the cell cycle, cell mitosis and chromatin replication, and the expression of SNRPD1 in malignant or the number of hyperproliferative cells is higher than in normal cells ${ }^{[31]}$. The high correlation with the cell cycle in SNPRD1 expression emphasizes that the cell proliferation of RTP patients may be more active. Similar to SNRPD1, SNRPD2 regulates mRNA splicing and is highly associated with Alzheimer's disease, which is similar to the KEGG results in group HR.

In our study, many enriched pathways and the effects of hub genes are related to apoptosis, which could be evidence of the hypothesis that the cause of reactivation leads to delayed virus clearance ${ }^{[7]}$. The immunity in RTP patients, the immune processes' neutrophils involved, endocytosis, NOD-like receptors and TNF pathways, as well as the immune cell function regulated by the hub genes, still require further research to explore their characters in RTP. Besides, the pathway of neurodegenerative diseases was significantly enriched, which makes the management and close attention of the RTP patients crucial.

However, due to the discrepancy in the theory of RTP and the small number of samples, our study has limitations. To make up these limitations, we expect further studies with larger samples to explore the association between each hub gene and the role of the pathways in RTP.

\section{Conclusion}

In general, using the biological analyses, we have explored the pathogenesis of RTP occurrence in COVID-19 from the immune mechanism and molecular level. With the analysis of DEGs, we found TP53BP1, SNRPD1 and SNRPD2 as hub genes that are considered vital to apoptosis, changes in immune cell function, cancer and neurodegenerative disease. We regard apoptosis as a key pathway in the persistence of the virus and its periodic release. Besides, the prognosis of RTP patients should be of concern because the pathway is regulated by hub genes in cancer and neurodegenerative disease. With these findings, the mechanism in RTP and related management measures for RTP patients would induce further clarity. However, aiming at deepening the understanding of the immune mechanism in RTP patients, we expect future studies to further explore these at the genetic and immunity levels. 


\section{Declarations}

Ethics approval and consent to participate

Not applicable.

Consent for publication

Not applicable.

Availability of data and materials

The GSE166253 datasets used during the current study is available from GEO database(https://www.ncbi.nlm.nih.gov/gds/).

Competing interests

The authors declare that they have no competing interests

Funding

Not applicable.

Authors' contributions

Xu-Guang Guo provided the original idea and constructed the article outline. Ke-Ying Fang, Gui-Ning Liang, Zhuo-Qing Zhuang, Yong-Xin Fang, Yu-Qian Dong, Chuang-Jia Liang, Xin-Yan Chen, were responsible for data analysis, table making and manuscript constructing. All the authors read and approve the final version of the manuscript.

Acknowledgements

Not applicable.

Footnotes

Not applicable.

\section{References}

1. Zhai P, Ding Y, Wu X, Long J, Zhong Y, Li Y. The epidemiology, diagnosis and treatment of COVID-19. International journal of antimicrobial agents 2020; 55(5):105955.

2. Rothan HA, Byrareddy SN. The epidemiology and pathogenesis of coronavirus disease (COVID-19) outbreak. Journal of autoimmunity 2020; $109: 102433$.

3."World Health Organization." WHO Coronavirus (COVID-19) Dashboard. https://covid19.who.int/. Accessed 30 Apr 2020.

4. Ren X, Ren X, Lou J, Wang Y, Huang Q, Shi Y, et al. A systematic review and meta-analysis of discharged COVID-19 patients retesting positive for RT-PCR. EClinicalMedicine 2021; 34:100839.

5. Zheng J, Zhou R, Chen F, Tang G, Wu K, Li F, et al. Incidence, clinical course and risk factor for recurrent PCR positivity in discharged COVID-19 patients in Guangzhou, China: A prospective cohort study. PLoS neglected tropical diseases 2020; 14(8):e0008648.

6. Yao MQ, Zheng QX, Xu J, Deng JW, Ge TT, Zhou HB, et al. Factors associated with a SARS-CoV-2 recurrence after hospital discharge among patients with COVID-19: systematic review and meta-analysis. Journal of Zhejiang University Science B 2020; 21(12):940-947.

7. Váncsa S, Dembrovszky F, Farkas N, Szakó L, Teutsch B, Bunduc S, et al. Repeated SARS-CoV-2 Positivity: Analysis of 123 Cases. Viruses 2021; 13(3).

8. Liu Y, Ding N, Zhou S, Chen C, Huang S, Lv Y, et al. Comparison of clinical characteristics between patients with coronavirus disease 2019 (COVID-19) who retested RT-PCR positive versus negative: a retrospective study of data from Nanjing. Journal of thoracic disease 2020; 12(11):6435-6445.

9. Song KH, Kim DM, Lee H, Ham SY, Oh SM, Jeong H, et al. Dynamics of viral load and anti-SARS-CoV-2 antibodies in patients with positive RT-PCR results after recovery from COVID-19. The Korean journal of internal medicine 2021; 36(1):11-14.

10. Szklarczyk D, Franceschini A, Wyder S, Forslund K, Heller D, Huerta-Cepas J, et al. STRING v10: protein-protein interaction networks, integrated over the tree of life. Nucleic acids research 2015; 43(Database issue):D447-452.

11. Lyu Y, Wang D, Li X, Gong T, Xu P, Liu L, et al. Continued nucleic acid tests for SARS-CoV-2 following discharge of patients with COVID-19 in Lu'an, China. PeerJ 2021; 9:e11617.

12. Gustine JN, Jones D. Immunopathology of Hyperinflammation in COVID-19. The American journal of pathology 2021; $191(1): 4-17$. 
13. Veras FP, Pontelli MC, Silva CM, Toller-Kawahisa JE, de Lima M, Nascimento DC, et al. SARS-CoV-2-triggered neutrophil extracellular traps mediate CoVID19 pathology. The Journal of experimental medicine 2020; 217(12).

14. Nunnari J, Suomalainen A. Mitochondria: in sickness and in health. Cel/ 2012; 148(6):1145-1159.

15. Gibellini L, De Biasi S, Paolini A, Borella R, Boraldi F, Mattioli M, et al. Altered bioenergetics and mitochondrial dysfunction of monocytes in patients with COVID-19 pneumonia. EMBO molecular medicine 2020; 12(12):e13001.

16. Glebov 0O. Understanding SARS-CoV-2 endocytosis for COVID-19 drug repurposing. The FEBS journal 2020; $287(17): 3664-3671$.

17. Li D, Wu M. Pattern recognition receptors in health and diseases. Signal transduction and targeted therapy 2021; 6(1):291.

18. Kim YK, Shin JS, Nahm MH. NOD-Like Receptors in Infection, Immunity, and Diseases. Yonsei medical journal 2016; 57(1):5-14.

19. Travassos LH, Carneiro LA, Ramjeet M, Hussey S, Kim YG, Magalhães JG, et al. Nod1 and Nod2 direct autophagy by recruiting ATG16L1 to the plasma membrane at the site of bacterial entry. Nature immunology 2010; 11(1):55-62.

20. Iwai-Shimada M, Takahashi T, Kim MS, Fujimura M, Ito H, Toyama T, et al. Methylmercury induces the expression of TNF-a selectively in the brain of mice. Scientific reports $2016 ; 6: 38294$.

21. Karki R, Sharma BR, Tuladhar S, Williams EP, Zalduondo L, Samir P, et al. Synergism of TNF-a and IFN-y Triggers Inflammatory Cell Death, Tissue Damage, and Mortality in SARS-CoV-2 Infection and Cytokine Shock Syndromes. Cel/ 2021; 184(1):149-168.e117.

22. Iwabuchi K, Matsui T, Hashimoto M, Matsumoto Y, Kurihara T, Date T. Characterization of a cancer cell line that expresses a splicing variant form of 53BP1: separation of checkpoint and repair functions in 53BP1. Biochemical and biophysical research communications 2008; 376(3):509-513.

23. Campagna-Slater V, Arrowsmith AG, Zhao Y, Schapira M. Pharmacophore screening of the protein data bank for specific binding site chemistry. Journal of chemical information and modeling 2010; 50(3):358-367.

24. Sundaravinayagam D, Rahjouei A, Andreani M, Tupina D, Balasubramanian S, Saha T, et al. 53BP1 Supports Immunoglobulin Class Switch Recombination Independently of Its DNA Double-Strand Break End Protection Function. Cell reports 2019; 28(6):1389-1399.e1386.

25. Tan W, Lu Y, Zhang J, Wang J, Deng G. Viral Kinetics and Antibody Responses in Patients with COVID-19. 2020.

26. Zhu JJ, Stenfeldt C, Bishop EA, Canter JA, Eschbaumer M, Rodriguez LL, et al. Mechanisms of Maintenance of Foot-and-Mouth Disease Virus Persistence Inferred From Genes Differentially Expressed in Nasopharyngeal Epithelia of Virus Carriers and Non-carriers. Frontiers in veterinary science $2020 ; 7: 340$.

27. De Biasi S, Meschiari M, Gibellini L, Bellinazzi C, Borella R, Fidanza L, et al. Marked T cell activation, senescence, exhaustion and skewing towards TH17 in patients with COVID-19 pneumonia. Nature communications 2020;11(1):3434.

28. van Eeden C, Khan L, Osman MS, Cohen Tervaert JW. Natural Killer Cell Dysfunction and Its Role in CoVID-19. International journal of molecular sciences 2020; 21(17).

29. Kim YD, Lee J, Kim HS, Lee MO, Son MY, Yoo CH, et al. The unique spliceosome signature of human pluripotent stem cells is mediated by SNRPA1, SNRPD1, and PNN. Stem cell research 2017; 22:43-53.

30. Kumar R, Verma H, Singhvi N, Sood U, Gupta V, Singh M, et al. Comparative Genomic Analysis of Rapidly Evolving SARS-CoV-2 Reveals Mosaic Pattern of Phylogeographical Distribution. mSystems 2020; 5(4).

31. Dai X, Yu L, Chen X, Zhang J. SNRPD1 confers diagnostic and therapeutic values on breast cancers through cell cycle regulation. Cancer cell international 2021; 21(1):229.

\section{Tables}

Table1

Details of the data sources for this study.

Table2

Upregulated genes and downregulated genes that meet the screening criteria in group CR 


\begin{tabular}{|c|c|c|c|c|}
\hline Gene expression profile & Sample collection & Sample genetic data included & Platform & Reference \\
\hline \multirow[t]{26}{*}{ GSE166253 } & peripheral blood mononuclear cells & GSM5066812 & GPL20795 HiSeq X Ten (Homo sapiens) & \\
\hline & & GSM5066813 & & \\
\hline & & GSM5066814 & & \\
\hline & & GSM5066815 & & \\
\hline & & GSM5066816 & & \\
\hline & & GSM5066817 & & \\
\hline & & GSM5066818 & & \\
\hline & & GSM5066819 & & \\
\hline & & GSM5066820 & & \\
\hline & & GSM5066821 & & \\
\hline & & GSM5066822 & & \\
\hline & & GSM5066823 & & \\
\hline & & GSM5066824 & & \\
\hline & & GSM5066825 & & \\
\hline & & GSM5066826 & & \\
\hline & & GSM5066827 & & \\
\hline & & GSM5066828 & & \\
\hline & & GSM5066829 & & \\
\hline & & GSM5066830 & & \\
\hline & & GSM5066831 & & \\
\hline & & GSM5066832 & & \\
\hline & & GSM5066833 & & \\
\hline & & GSM5066834 & & \\
\hline & & GSM5066835 & & \\
\hline & & GSM5066836 & & \\
\hline & & GSM5066837" & & \\
\hline
\end{tabular}


Up- $\quad$ DUSP11,KANK1,LZTR1,EEF1A1P19,VMAC,FOSL1,CTSZ,IFITM5,ARHGEF1,B3GALNT2,HLA-DRB5,MCM3,H3F3B,SLC25A6,CD79B,HLA-F-AS1,TRBI regulated DT,PQLC1,GNAL,ACTR3,PIM2,ZDHHC4P1,SNORA9B,MYO6,ALOX5AP,HSD17B13,AOAH-IT1,TBC1D16,WASF2,RTP4,SIGLEC10,CD52,HSP90B1,GIA DOA,PGLYRP1,SF1,HMGN5,CASP17P,SLC4A1AP,DNAJA3,NADSYN1,SLC25A36,CD48,CD200R1,SLC39A13,ARHGEF10L,CEACAM8,RBM12B-AS1,I AS1,FCER2,SCARNA9,CLCF1,RNF181,PECR,GAB2,SCARNA8,SH3TC1,SLC25A40,SMAD1,VBP1,SMG1P5,RASSF2,DOCK9-DT,STK10,MAP3K12,ME AS1,RDH14,CD99P1,SEMA3F,NUDC,KRBA1,PYGL,TARS2,ZNF410,HSP90AB1,CAPG,LYAR,MOB2,RNF146,HNRNPA3P15,CALU,B3GNT7,Z83843.1, DOB,SERTAD3,RN7SL329P,SAT2,XBP1P1,TIMMDC1,UBALD1,APOL3,NMRAL1,APTR,CCDC9,MALSU1,WDR81,FCN1,TXNDC5,RASA2-IT1,SMTNL1 AS1,MAPK8,GIMAP8,LRCH3,PLAGL1,ZMYND8,APOLD1,GASAL1,OPCT,DICER1,NBPF1,CASTOR2,UACA,CARD9,KAT2B,MARK4,COLO,OGFOD3,TR 805PCRELD1,AC103810.5,IFI6,LMNB1,DNHD1,XRRA1,ABHD4,HACL1,CCT6A,KLHDC8A,EIF4EBP3,EDF1,PCF11,WASF1,TBC1D30,SLC27A4,TMCC DT,USP15,TRAJ24,Z98752.2,NAP1L4,EFCAB2,ZNF18,MAP4K4,C1QL3,WDR18,CDC14B,RAB6B,NPM1P25,PPM1L,RWD' 3,CIAO3,COPB1,MYBPH,I 1,JRKL,PZP,CDK3,TMEM161B,MIAT,GBP6,UBL5,GTF2IRD2,WAPL,MRPS5,PARS2,ADD2,FRG1,CREBZF,PARP14,STK17B,CUTC,WDR11-AS1,MED2\& AS1,MITF,PRR16,ILVBL,FDPSP3,FCRL3,SLC1A4,THOC2,AIMP2,KCNK5,TRIM51BPZSCAN25,ENTPD2,TMEM245,LPPOAS2,ATP5MC1P3 TBCA,ZN 59P,CDK10,COX6A1,AC243960.3,ZNF318,KCNH3,OXSR1,PDF,PCNPP1,PHF7,DEFA1B,MICAL3,TLN1,COPS7B,LINC01465,SNORA63C,PRKD2,AKR AS1,NHSL1,GTSCR1,YDJC,TMF1,CDK20,MBD1,TMEM150A,POLR3D,HERC5,ZNF69,NFE2L2,TMEM120A,TMEM126B,SMIM10,IGHV4-59,COCH,M 807P,GSDMD,TGIF2,MRPL46,DEDD,TMEM158,STX1B,TEKT2,MRPL33,TBC1D2,OR7E140PCLP1,RBBP4P1,CKAP2,AATK COMMD9,PDK2,ZNHIT1, AS1,SLC25A42,RNU6-623PCYCS,PIK3AP1,MPV17,AMDHD1,NARFMCMBPAC139530.1TCEA1P4,ITIH4.PRR11,GJB2,NUDT17,SMG9,CASD1,NSL AS1,SMAP1,CD27-AS1,TGS1,L3HYPDH,SLC28A3,DCST1,NECTIN4,FRG1DP,PCGF1,CLIC5,MPST,HEXIM1,,SIKE1,BBIP1,TRBV12-3,AL096865.1,CLE 275P,FBLN2,EOGT,HEATR9,CANT1,POM121B,CCSER2,NDUFAF1,WRB,UGP2,IMPA1,CWC15,COX7B,AGAP9,APOL6,TAZ, SPARC,CHMP4B,UQCRQ,IN AS1,ZBED1,PMAIP1,MRPS24,AKR1C3,ENTR1,RAD54L,MICALL2 SLC25A34,PRRT4,ATXN2,IKZF1,FKBP1B,CBY1,SMPDL3A,OLFM1,STN1,TRAF7, A DT,ACSS2,C7orf26,COL4A3,ELOB,POLR2H,ENKD1,WDR91,ATP5ME,COP1,RAPGEF1,VPS26A,ZNF573,UQCRFS1,DHRS4L2,MRPL47,MDM1,XCL1,I AS1,FKBP5,XPNPEP1,NTSR1,ACO25917.1,AP1M1,TRBV10-2,MMP17,NDUFAF4P1,FBX046,CEP120,CASC3,POMGNT1,WDR3,CYB5B,SLAMF6,ZN AS1,CD93,NSRP1,NCAPG2,TENT5A,PTPRC,AKAP12,AL662907.1,C2CD4D,HMBS,MIER3,DDX56,ZNF582-AS1,MRPL54,MY019,TPGS2,NUPL2,CBI AS1,SLC1A7,SPRY3,CLTB,AGAP1,SPATS2,AL645939.2,ZNF165,SPEN,NAA25,CCNL1,HECA,PRRC2B,AC003072.1,GATAD2B,RRP1B,CYB5A,UBE2D 4,DISC1,NBEAL2,CCER2,AQP9,RPL26P9,AL355987.4,USP14,PMS2CL,MS4A4A,IP6K1,TRAJ10,TIPRL,ZBTB25,SNORA80B,DCAF13,CHST15,TYSN AS1,RCCD1,CAMK1D,RRBP1,TRPM7,SMARCD1,AES,ODF3B,TPR,ASB14,MEAF6,GP5,USP22,IL 12RB2,CLC,MRTFA,GHRL,TUBA1A,NOLC1,ALDH3B 6,COASY,DEXI,MICU3,TRAPPC10,COPS9,TRIP11,IARS,IPPK,KDM2B,AC079305.3,C4BPA,BOK,SIGLEC8,MORC3,CD36,SNRPD2,ZDHHC11B,CITED2 57P,EID2,ARL16,FBXW8,ZNF26,PHF21A,ACTR6,NCBP3,C1orf109,BORCS5,CYHR1,GLT8D1,MECP2,MRPS17,AC007342.6,LINC00102,TRGV5,CRIP AS1,TYW5,ADGRE2,MTMR3,DSC2,ERO1A,XBP1,OMG,ALG9,ATPSCKMT,THAP9,SMPD4P1,MAN1A2,EXOG,SPTBN1,PTGIR,SPINDOC,DNTTIP1,ST AS1,AC132192.2,KMT5C,SPRY1,KCNRG,REX01,CTC1,SOS2,TDP2,MED25,CHCHD2,ADRM1,C6orf203,MMAB,TP53TG1,LRCH4,BEX1,HSPBP1,AN AS1,KCNMB4,RNF214,NIPAL2,FLI1,ANKRD54,OSBPL 9 COX6CP1,JMJD6,MTF2,KNL1,MCM4,AL 161757.2,FCMR NCKAP1,GNMT,ORICH1,VPREB3, 4,AP001266.2,SNX18,LINC01678,SYNPO,DNASE1 L3,CUL1,NPC1,UBE2Q2P1,PRCC,ZNF672,FSCN1,UCN,SLC30A1,ID1,DPP7,TDRD 3,GNGT2,PDZD 603P,SLC25A12,FM05,PCYT2,TMEM81,EXOC3-AS1,UCKL1,PSMB9,MTG2,SLC17A9,KLKB1,DPYD-AS1,XPNPEP2,RBM22,PDXP,WIPI1,BCL9L,ARS AS1,UPF1,LNCAROD,PER3,DPAGT1,HDDC2,PADI2,SDAD1,EZH1,AC025164.1,CRTC3,SOX12,HERC6,EEF1E1,SNRPCP3,SENP7,CETPNDUFA13,TR/ AS1,SMPD1,MPP5,SNORD12C,ARL15,TRIQK,CMPK2,CHRNA10,GFI1B,SPG7,IGHJ5,GRIN2C,ZNF554,DTX4,ACADVL,THRB,HSPE1P11,AC244157.: IT1,AC011450.1,BNIP3,C12orf65,MYDGF,METTL7A,AP005019.1,ADAP2,C9orf16,DPEP2,PGBD 4,WBP2,MAN2A1,GPER1,SKAP2,MICA,ARRDC2,AK 13,LHPP,LINC02453,AC016999.1,STMN3,POU5F1P3,CARS2,LINC02356,ATRIP,NEMF,IP07,COPA,AC010326.4,ALG13-AS1,ERMP1,SNX11,PKD1,GC AS1.FAS RCL1.EMC2 OR2A20PAC008115.2 EID1.KATNAL 2 AL133406.2MIR302CHGAC138393.2 PEG13.DNA2AC087741.2 TBC1D23,AL596202 IT2,PIGG,PFKFB4,LAT2,ZDHHC1,TNRC6C,SH3YL1,RF00560,AC004477.3,ABCC13,TTC17,LDHAL6A,COMMD2,HSD17B7P2,MLX,EPM2A,MINCR,N IT1,SPTAN1,GYPA, TRAV5,PPP1CA,GMEB1,NDUFB11,EIF1AD,ERP44,SLC18B1,CTSO,ZSCAN26,MIR3198-1,RNU6-662P,HDLBP,NAMPTP1,CLCN5,C 625PGBP1P1,TTC22,RPL30,SNORD124,MSANTD2,AC006111.2,RAI1,BBS7,ELAC2,PCOLCE,TRAV20,PRR13P2,LSM12,ABCA1,C1orf35,HLA-DPB1 DT,TLR5,KLHDC8B,EXOSC4,UBA52P5,ST6GALNAC4P1,APOBR,EMB,CLNS1A,MORC4,CADM4,REX04,RAB18,AC025171.3,AC087854.1,TIMM23B AS1,MED 27,BCL 10,TMED6,NOCT,MRPL23,INVS,COG3,CUTA,PAGE2B,PARL,PPP4R1L,MRPL51,POLR1A,FCGR3A,CCDC65,GDF11,AC147651.1,ND 6,AP003498.2,HSD11B1L,NDUFS2,ZNF853,LINC01136,DNAJB13,ASB13,HHEX,BTN3A1,GAS2L3,AL022157.1,D0P1A,AC012307.1,AL442003.1,T AS1,A0C1,UCP3,ZBTB21,AC040934.1,WDFY3,COPZ2,AC027575.2 MED31,SIPA1L1,ACBD5,PPIAP22 GCSHP4,ANKS3,MRPL 55,PPM1K,TMBIM6, AS1,AF131215.6,FRYL,ADARB2,CDH26,NCAPG,NHP2,ZNF607,R3HDM2,LINC00482,UPB1,AMMECR1L,ZC3H6,EVA1C,C3orf33,PLA2G6,NRDE2,TR AS1,KIFC3,EMBP1,MTX3,GIPR,AC023509.3,REPS1,TRAJ16,NVL,AC116366.1,SLC22A1,LINC02265,PVR,ADGRB1,AVL9,EBPL,COQ10BP2,NCAPD3 AS1,NUP210,TMEM88,FAM161B,AC027176.2,F0082796.1,IGHG3,SRRM1,MAP3K10,C16orf87,TDRD6,FAM234A,SNHG14,POLD4,PPIP5K2,COMN 479P,CHST2,EFL1,C19orf44,MVD,SNORA38,FAHD2CPTOB2,FAM98C,E2F2,AC008753.3,USP31,STK39,IFT52,TMEM242,CCDC200,ERLIN1,UPK3A AS1,AASS,GTF2IP12,SIRT6,FAM49A,ARL2BP,PATZ1,PTAR1,HIST2H2AA3,YBX1P2,AC008750.2,WDR19,NCAPD2,AGAP10P,TCTEX1D1,AL354877. 106P.PIGZ,YWHAG,PIEZ01,LINC00847,RF00154,AL353622,2,IGHD,CYP4F29PSPDYE12PCLEC9A,PEX10,CRYL1,BUB1B,ERCC4,HERC4,SIK1,CA3AS1,SLC2A9,RRP12,ATP6V1FNB,MFGE8,TMEM254,COX20,PPARA,LILRB1,CCDC92,HIBCH,KRBOX4,GALNT2,SNORA40B,GARS,TAS1R3,TRMT10, 1,BCL2L15,ANKRD52,AC008035.1,AC090181.2,ZKSCAN5,RAP1GAP,DPY19L2P2,PWP1,SNORD72,MINDY3,CCL2,CRYZP1,YIPF2,TRAV38-2DV8,G DT,AC244197.3,MEIS1,AL158163.1,ARL14EP,AL031963.3,SUCLG1,ZUP1,C8orf33,CDC14A,HDAC4,EPHX1,LINC01474,NTNG2,EPB41L4A,ENKUR,N 40P,NEIL1,KRI1,PRKY,EVI2B,COX14,BCDIN3D,SFXN5,AC009041.2,LINC01988,GOLGA8B,ITPR3,TRAJ5,RF00573,AC006946.1,XKR3,LINC00888,LTI AS1,DGAT2,AC022960.1,LEXM,CLPTM1,CCDC82,Sep_9,AC037459.3,THBS4,POLE4,FITM1,AC124016.2,TEX45,SMDT1,FUNDC2,MPLKIP,LINC023 AS2,AP000866.6,HP1BP3,MED6,PIGU,LGALS12,C12orf43,AC006960.3,NLRC5,AL590714.1,AC012313.10,CCDC106,TRAJ21,AC117503.2,ZNF52ع

Down- HBEGF,CXCR1,HBD,TARDBP,FTLP3,FTH1P16,MTRNR2L2,HBB,TNFRSF12A,GAPDHP14,LARP1,BCL2L11,CXCR6,C5orf15,HIF1AN,NANS,CXCL5,TC regulated AS1,SH2D1A,WWC3,ACSL3,PFKM,CXCL16,MOB4,CD84,MAP4K1,LPCAT4,PGM2,ARF3,CCR12P,FOSL2,MMS22L,RNF122,PILRA,IRF4,IREB2,LGAL؟ 14P,MTC01P11,B3GNT8,FOX04,H1FX-AS1,EME2,TRG-AS1,ATP1A1-AS1,ARF4-AS1,KCTD5,P2RX1,RAB24,PHF12,SLC35E4,EHD4,TSHB,GSTP1,SC 646P,TIMP2,PTGFRN,ABCA11P,TPPP3,ELK3,ATP13A1,SMURF2,00SP3,ZNF467,KLF5,GPR89B,STX3,CRIM1,ZBED3,RBBP4P2,ZNF845,MPEG1,ZB 50P,HSPA1A,SPIDR,TRAJ3,GPR75,WHAMMP2,EMSY,MYADM,CHPF2,Z97180.1,ZNF677,PPP1R8,ODF2,VIPAS39,SLC25A17,CCDC78,TDP1,ADAM AS1 ZFR CHST8,SMC1A,BRD3 TSC1,SPSB2,FTLP2 ZNF79,SLC24A3,HSD17B1,GRN,ITCH,MIR7854,GPATCH1,ZNF655,NFIA,FNTA,MIPEPCORO2B 487P,PDE4D,ISG15,AC004854.2,ZBED4,ZNF185,AC016949.1,MIR6763,PTPRJ,ARL6IP4,YPEL5P2,ZNF33B,HOXB2,TFDP1,LONRF3,ACSL4,WDR37 AS1,MIR219A1,ANAPC1,NR2C1,PLD2,GK3P,TMEM168,TRIM27,UCK1,ARID4B,PLEKHG5,PARPBP,TANC2,TAS2R20,RNF216-IT1,AC010737.1,PSMC 79PPNPLA6 POMPAL 591846.1,NME3,TPRA1,AC093627.4 SOCS6,SPICE1,HMGN1 HOXA2AL 590609.3.CXCR5,CHMP2A GRK6,EFCAB13,PSMA1, 1,ABALON,KIAA0355,DRAP1,SLC2A3,SF3B2,PHF5A,NHLRC1,STXBP3,CREB3L4,XRN2,FTL,AL139041,1,HIVEP3,GBGT1,RFXANK,SERAC1,GNG8,T 1266P,LAMB1,AL 122001.1,CCDC136,P2RY12,RPS20P14,MFAP4,MIR3936,AC092053.2,CCDC189,SPIRE1,EDRF1-AS1,UPF3A,ZNF257,APAF1,CLD AS1,BRAT1,SNORA12,INSR,TEFM,RN7SL4P,C12orf29,SLIRP,LILRA4,PRKAG2,AP001962.1,CAMKMT,RNA5SP111,SIL1,GPATCH4,LOXL1,AC132938 1310PHOOK1.LINC00677 HHATBCCIPVPS52 NIPSNAP1,GPRIN1,FBXL13,AC0255942,AAK1,AC021087.1,KPNA4 MIR4740 CTLA4,SYT2 CHAD,AC 8SN1,AC093826.2,ADCY4,GEMIN4,KRT17P8,NFATC2IP,RAB11A,AC006460.2,DNAJC16,PJVK,RPL32P3,AL009031.1,SNAP47,UBXN8,AL121820.2 40,MIR373,C5orf66,COX10,L3MBTL1,AC002401.1,ZNF75D,TRGV4,EIF4E3,AC103706.2,MIR4668,Mar_5,ITGB1BP1,LSM10,PLLP,SNORD100,AC06 DT,FBX032,BX679664.3,AC009318.1,WFS1,PMS1,RHOU,PVALB,TCHPRXFP4,CNTNAP1,ARSK,FADS2,MT1XP1,FOXD2-AS1,AL031777.1,PCCB,RF AS1 ROCK1P1 AC096733.2 AC007969.1,EIF4G2,RF01225,AL138995.1,RBP5,RPS18,RPELINC01402 C1 orf146,ZNF502,AC022182 1,ZNF830,MIR: 188P,POLR2M,ZSWIM7,TNFAIP1,RGS10,AC145098.1,RNA5SP345,AC139100.2,CTU2,TMEM87A,ZNF641,LINC02604,AC009299.3,AC034222.2,PI 249P,ZNF140,AC116348.1,FGFBP3,AC245052.6,AL135925.1,PGBD1,SLAMF9,AC108134.1,SNORA22B,RNU7-180P,AC079035.1,AC073641.1,PSM

\section{Table3}

Upregulated genes and downregulated genes that meet the screening criteria in group HR 

DPB2,RNF144B,GPR25,RPL23AP7,ARL4D,HSPB1,FOSL2,IL1RAP,PDIA5,CLIC3,SHMT2,ZNF600,PPP1R2,HSP90AB4P,SCARF1,UBE2V2,RBM43,USF 658PRNF169,PLCB1,ARHGDIB,MIR26A2,KLF3,NRADDPTBC1D14,IRF4,RBM4,DNAJB12,FES,EEF1AKMT1,TM9SF1,PTPN2,ETFB,DD0,MOB3A,AIFI 1024P,MARF1,SIGLEC11,HDGFL3,PTGS1,JPT2,SPRY2,CFH,LINC01629,IRGQ,GABARAP,MORF4L2,ARID 5B,EIF3J,PQLC1,BCAS3,DEF8,EHD4,CIB3, IT1,CLK3,GLUD1,NARF-IT1,Z83844.2,COX8A,HES4,GOLGA2,IFNGR2,PPIG,PPP2R2B,EIF3B,APH1B,HES1,PPP1R2B,DUSP12,ARF6,LYAR,GNAI3,KHI AS1,ZNF486,CSF2RB,PLK1,NAP1L4,CCNH,TPRN,STK11,PRG4,GAB2,RAPSN,MTC01P11,ITGAX,FUT10,SH3BGRL3,GLUL,REM2,ZNF487,CNPGIN1 AS1,FUCA1,TRIM58,EID2B,MAPK1,HMGB1P14,ZNF322,SRGAP2B,Z84480.1,PLEC,SLC4A10,CBFA2T2,VARS,BMP1,ARMC1,CD8B,PLEKHA1,ATP5 652P,CFAP36,TBK1,OXLD1,MBD5,LMNTD2,STIM2,KPNA6,ZNF300,ATRN,EME2,BLZF1,ARL6,NDUFB2-AS1,AMIG01,TAL1,AJ003147.3,ARHGEF34 AS1,GHRLOS,NCOA2,TRIM51BPMAPK7,TRAPPC6A,SLTM,CTSC,DNAL1,MRPL13,TBC1D32,FAM57A,ECH1,CLINT1,AP3B1,XP07,MICOS10,TRAJ3 AS1,AC095057.3,ZNF324,NXPH4,CBX8,ZNF33A,RNU1-27P,PPP4R3A,BICRA,PAIP2B,BAG5,PSMC6,CNPPD1,CSNK1A1,TTK,GATAD2A,SH3TC1,ME ATP6,CCZ1,SREK1,MDFIC,MRPL50,HSPA1A,KARS,TCP11L1,KPNA5,YWHAZ,TPRKB,BTLA,PTPRK,COX7B,ZBTB40,NCEH1,WBP11,STRIP1,SNORD AS1,MRPL33,MMP17,TMEM116,SPON1-AS1,THEM5,TTL,ABHD15,GNA15,PSMB6,TLK2P1,CHIT1,PDK2,DRC3,NUP37,AC100810.3,ECI2,CAMSAF 646P,RAB39A,RWDD4,SF3A1,NUDT4,SLC9A8,FBX08,CHORDC1,MTMR9,FZD2,H2AFZ,PRKDC,EPB42,UQCRFS1,TRGJP1,MRPL47,ZSCAN18,ENTF DOB,TFDP1,AP1G1,ZNF185,PPM1L,FAHD1,JAM3,DHRS4-AS1,AARS,PRKCZ,VWF,AGA TMEM198B,MRPS24,MRPL39,PPIAP40,ETV6,TRAV12-1,ZI 4,NR1H3,FOX01,BCL2A1,TRIM9,CDCA4P4,RBX1,CDC7,ATG16L1,VN1R81P,COMMD9,AC012615.4,GPAM,ZBTB20,AC079305.1,CIAO3,JPT1,LRRC AS1,PSMD7,VENTX,PRPSAP1,CTSW,SMIM15,STN1,TRIP11,AC243829.2,TENT4B,RBFA,CENPBD 1P1,CAPNS2,XBP1,TRGV6,IFT80,SHISA5,IDH2,C 79P,NR4A2,BFSP2-AS1,AP3S2,METTL21A,DDX56,TBC1D22B,SETX,LINC00680,ICMT,BTF3P12,EDEM3,ACTR2,GINS2,KIF3B,R3HCC1L,RNU6-45P,I DRB1,NUPL2 TRAJ41,UPF3A,TMEM256,PIGC,VAMP3,PAG1,CCDC126,FAM118B,MYD88,MGMT,WHRN,AUNIPPPM1M,NBEA,BSPRY,PNO1,RALBP AS1,SCGB1B2P,STRADA,SEPT14P4,ZNF276,ARF4,TBL2,PHLPP1,LRRCC1,CWC25,TTYH2,MAEA,MMP28,BLVRB,CCNJ,DRAM2,SPAG1,GADD45G, 662P,NELFA,CASP8AP2,HCFC1-AS1,TIMM9,MED12,TBC1D3L,ABHD12B,CXorf40B,SVIL-AS1,INSIG2,PTK6,EPG5,SHISA8,CRY2,CKAP2L,TRAPPC1 AS1,MED24,MECR OVGP1,TBCCD1,TLDC2,CDC42P4,SMARCD1,TIA1,TRIP6,STK3,PPP6R1,SPINT2,ITGB5,POMGNT2,SLC19A2,RBM15,IFI44,BEX AS1,ATP10A,CCDC18,CYP2U1,BRWD1,AKAP9,LTK,MTC01P12,B4GALT4-AS1,GPATCH11,LSM4,PDSS1,0XSM,GHITM,NCKAP1,MRPS17P5,DDX3 946P,KCTD3,TDGF1P6,SUN2,STK25,FBX030,MPHOSPH8,ZFC3H1,CUL4A,BISPR,EMC2,PDCL3P4,SKA2,IRF2BP1,TMEM44-AS1,TMEM159,DDX5 541PTRAV23DV6,SPNS1,NEDD8 ZFAND3,PIGB,CITED4,CRBN,ZNF529-AS1,LIMS1-AS1,ZNHIT1,YPEL5P2,DBF4,SNORA59A,ALDOA,POLR2F,TNFS AS1,AL162430.1,SLC40A1,LSMEM2,PFKFB2,AMN,BIN3,FITM2,MINDY1,ZNF581,BCAS2,DIS3,HID1,C5orf30,SLC25A14,ZFAND2B,VAT1,MRPL12,T 762P,NODAL,SPTBN5,ANKRD42,AC040160.2,ZNF525,CTSE,LY6G5B,HTR7,NDST1,VPS37D,TCF7,HIVEP3,POLA1,ESD,CCND2,ETFBKMT,VWA7,ZN 1,C19orf54,TMEM80,ZNF44,RBMX,SLC25A39,CNOT9,GPR183,NACC2,HEMGN,HEATR5A,LRRFIP1P1,BTBD6,PSMB9,PRKCI,ELOCP31,FAM151B,F AS1,HNRNPA1,CLIP4,SLC30A1,HAUS8,TDRPZNF527,BCL9L,AC004889.1,STK19B,SLC6A16,MICD,ERAP2.PGRMC2,ASCC1,CNOT4,BCKDHANPM AS1,TNIP1,CHD8,UXS1, HADHA,PSMB2,ZNF770,ZBTB6,DFFA,DCUN1D2,CADM1,NOB1,SH3GL 1,FAM210B,TRIM28,VWCE,POLR3H,TMEM134,SPC DT,ATP5PBP1,ERFE,IQCG,EZR-AS1,MRM3,ZBTB7A,TRAV10,CDCA3,CDK2AP2P2,ZNF41,AL357078.1,AMZ2P1,SNORA31,ZNF227,MRPL45P2,DLG 729P,HMGB1P20,AANAT,CCDC25,COX11,SIK2,GPR65,KCNMB4,FBXL17,KCTD20,KYNU,TAPBPL,USP12-AS1,LAMTOR4,ALKBH4,PPP3CC,SLC25A AS1,TRIM39,PDP2,ISY1,SHMT1,DISC1,GFI1B,OMG,ZBTB49,ZNF121,DCAF1,CALM2,IFT43,IMPAD1,EHBP1,GP5,KLHL36,AC113935.1,NDUFB11,KI IT2,ZFP90,SNX18,ZW10,ADHFE1,CHST8,AMD1,GRAP2,USP13,GAA,SPIRE2,HSPBAP1,POMGNT1,AASS,TOB2,DNAJB14,PMS2,VCPKMT,SPRY3,A DT,MRPS16,JRK,MALINC1,NDUFB8,LCN12,ASCL2,HABP4,PNKP,DCTPP1,MRPL24,AGRN,MGPFUOM,HIRA,FHIT,TARM1,COMMD4,EXOC3-AS1,REC 4.PARD6A,SLC26A1,NCR1,MYDGF,LINC01881,SLC7A60S TAF12 NKD1,TMEM123,AC007386.1,NRIP1,CPNE2,ITIH2,USE1,NUMA1,MED22,PRKAG: AS2,CPSF6,MAP3K1,SOD1P3,PLA2G4C,ZMYM6,LRRC14,MDGA1,ACVR1B,C6orf52,FAM204A,ACP5,MTPN,AC090229.1,ADAP2,QRICH1,PITRM1,F 701P,PCNX3,HOXB4,ZNF280C,ATG14,FAM193A,AC008966.2,HMGB3P22,MPP5,KDELR1,RBAK,PPIP5K1,MHENCR,UCKL1,RNU6-574P,UHRF1BP1,: AS1,POP7 TOP3A,SPRY1,ACTR8,PDXDC1,EMD,UMAD1,ZNF81,ARSD,CPLANE2,TRMT112,TC2N,MFSD1P1,EX0SC6,PMS2P3,PBDC1,CTNNA1,SY AS,ZNF230,MRPS17,SUPT3H,UBL3,AC021242 3,C10C,DNAH1,TAOK3,FAM183BPCOR06,AC067945.1,SLC18A2 SNRPD1,SPCS1,GSDMB,CHST1 AS1,CDA,OAS3,BCLAF1,YPEL3,ARHGEF19,BZW1P2,AC073172.1,S1PR1,PPP2R3C,EMC7,TMEM71,CYB561D2,ARPP19,HSD11B1L,PRPF31,FAN1 AS1,PNISR,SH3YL1,LZTS2,AC125437.1,PAGE2B,ARSG,AASDHPPT,ZDHHC19,EIF3D,CASS4,RNY1P11,PKHD1L1,CIPC,AC026412.1,RRN3,SEPHS1 686PFARSA,KIF20A,AK9 ZC3H12A_ANAPC16,AL138963.1,AL359183.1.PGAP3 CETPFASTKD5,OTULINL,ITPR2,ENKUR ZFP82,AL050343.1,RNF24 59P,FRYL,AC087241.4,SOGA1,TRIM65,ZNF100,APH1A,MSL2,TRMT10A,AC011465.1,EFNA4,FAM122C,TRPT1,SETP17,ZNF518B,RCOR3,DCPS,A7 1300P,FADS3,ADORA3,CLOCK,MRPS31P4,C1orf147,ANAPC11,DEPDC1B,FZD 5,TMEM117,EEF2K,MFF,AC026748.1,SPDL1,EMC8,EIF5B,ATRX,DB IT1,PLCB3,PMPCA,SEC61A1,MIR4514,PTPN18,TRAJ57,FOXJ3,TMEM191C,CUBN,EIF4EP1,LINC01474,MNDA,TAF1A,SQOR,MYOM1,AC119428.3,

Down-

HBEGF,HBD,HBB,MOB1A,IFITM5,FCH01,SH2B1,CSGALNACT2,MALT1,CXCR3,NANS,SPAG9,SGPP2,SLC25A6,FPR3,AL627311.1,CXCR2P1,AQR,P1 3,NAP1 L1,OLA1,AL627309.7,ST3GAL6,OGFOD2,IQSEC1,LYPD3,MELK,FLT1P1,ADAM9,AC012368.2,PGLYRP1,SUCLG2P2,CLCF1,UBE2E3,AL3919 1010P,CREBL2,ST6GALNAC3,PTPRE,RALGAPA1,PALD1,AC108673.3,MEGF9,ANTXR2,CNN2P9,ZNF132,BCL7B,PAF1,LPCAT1,PTGDS,HSD17B13, 482P,PYCR1,NLGN2,LAMTOR1,TMEM260,AD GRE3,TP53BP2,NRF1,IL16,HAPLN3,DZANK1,RBM3,HEY1,RGS12,SH3BGRL2,CASP9,BAZ2A,DENND 977P,RER1,TCTEX1D2,PAD I6,RDH10,TRAJ35,CALR,GDPD1,ANP32B,FNTA,AC007278.2,APOL3,CEACAM6,MIR580,TXNDC9,DGCR11,ULK4,MMP9, AS1,IL 18,PNMA1,TPP2,AC018926.1,TDP1,AL513327.3,FAM157C,ISM1,HCG9,MBTPS2,AL049840.5,CARD9,ZBTB38,AL390774.2,URI1,AC020908 IT1,AC008735.4,THAP1,AC104763.3,MPIG6B,TRDD2,RPL37AP1,IGF2R,ZNF282,AC104411.1,ERN1,PRADC1,AC018946.1,R060,RAB6B,MIR8064,I AS1,HBQ1,CCDC194,PCNP,DSTYK,RASSF2,CALM3,NCOA4,TTC16,FBXO9,AC005682.1,RF00593,MIR4269,RPS7P1,AC104564.3,CD5,MIR4324,SU 7,KDSR,AC100847.1,ZNF586,CDK13,AC011374.2,JRKL,C4BPA,MIB2,NINL,SLC27A4,MCUB,KIAA1522,LINC01004,MKRN1,AC004687.2,AL354744. TG,MIR548AK,HIPK1,RAB2B,AC007365.1,FN1,AC007342.9,YIPF1,SLC25A26,AOAH,ACSL5,MS4A14,ARG2,GPR34,FAM160A2,MASP2,GIHCG,AL3 1017P,ATP5PO,NT5CP1,TSPYL1,AL732372.3,TMEM208,AC069287.3,RNY4P7,FLNA,AC066613.2,PTPRO,UBE2G1,TP53I11,VILL,SSBP2,TRAJ45,E 59P,AC093010.2,ITGA9,AL031432.3,SETDB1,PPP1R14B,KHK,AC067838.1,SRSF8,TMEM17,TBC1D15,BET1,PTMAP12,DBR1,AL139353.2,SLC30A 1275PAL 353764.1.CCR10,IL1B,FEZ2,AL590609.3,APCDD1,AC010326.4.ME1,EXD3,ZKSCAN4,Mar 7,HIST1H2BFCPLANE1,AC009570.1,EIF4A2,A 50P,ZNF746,AC055839.1,NTSR1,HIST1H2AH,ZNF680,AC010531.5,RHOBTB2,AC005154.2,CCDC127,POLI,AC130454.1,WASH5P,RDH13,ASPSCR DT,PACRGL,SRP68,STARD4,AC005261.5,TIAM1,APC,AC013244.2,RPL26,AC092634.3,TRAV39,ZNF701,HIST1H2BI,SNORA24B,STARD 5,DHX57,A 223P,NUDT17,ING2,AC091185.2,BBIP1,DAPP1,FAM215B,CASC3,FADS1,AC008731.1,AC005332,4,UBL7,SLC25A25,AC244157.2,AMZ2,AC021087 29PUBE2G2 MIR4484,RNASE2,NSUN7,DHX30,EPS8L1,TRAV25,AC006946.1,OSGIN1,AL591848.3,SLC20A1,RUVBL2,SIRPG,BLOC1S5,PIP4K2A,SL DT,PDLIM1P4,STAMBPL1,MIR5190,CERK,CAPS2,AL139022.2,CLTB,BNC2,GOLGA2P10,ZNF93,AC099811.6,MTMR12,AL031963.3,LINC02207,AC 1,RNU4ATAC,RHEBL1,LINC01063,RBP5,MIR5690,AL512791.1,AL031846.2,AC090607.1,UBE2H,ADCY3,NPHP4,AC010654.1,RSAD 1,STEAP4,AP0C 141PMYCT1,MDC1,RF02154,RGL2,PCYOX1L,AC021106.2,AC018665.1,AC005776.2,AC008753.3,EZH2,OSGEPL1-AS1,MIR6731,GBP1,AC099063

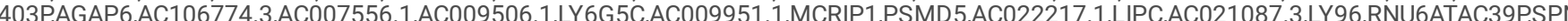
19,DYNC1H1,AC074032.1,AC009121.1,NENFP1,RN7SL600P,AC112173.1,SNRPA1,GAN,AC005154.4,AC008115.1,DNPEP,AL445584.2,AC099548.: AS1,AC006960.1,UBR5,NLRC3,AC104010.1,TMEM52B,PILRB,NOP53-AS1,AC074132.1,RENBP,AC005391.1,RAB5B,AC132942.1,RF00285,GK-AS1 AS1 CYP2T1PCNOT7 TTLL11,AL391832.3AL162377.1.CCDC144B,AC125232.2BTBD11,GABPB2 SNORD 98,AC010615.1,NSUN3,AC008753.2PF RNR2,AC022150.4,AL 022328.2,AL513365.1,AC108718.1,SS18L2,RNA5SP78,AC136475.10,ZNF32,PDCL,RFX7,SOX12,SLC27A3,RPL34P22,AC00 5,SFTPD,AP002360.1,FANCF,RF00599,AC092053.2,SC5D,AL 136141.1,DNAJA2,PRDM1,AC013356.3,AC009159.3,AC010737.1,STRADB,AC00424 AS1,TRIM14,RUSC1,ABCA1,S1PR5,SRP54-AS1,AL121845.4,SLC4A8,AL031710.2,HECW2,HEATR6,RPSAP58,GIMAP7,CRACR2B,AC025171.1,GOL AS1,ACTN1,LINC00945,HSD17B7,AC008680.1,TRAJ54,MIR646,AL355480.1,ANKHD1,SMIM10L1,ASMTL,RNA5SP201,RNY3P8,ABHD2,ZCCHC24 23P,AC005332.6,AP4B1-AS1,AL138724.1,MTCH1,AL162615.1,DGAT2,POLR1B,NHLRC1,RPP40,AKT3-IT1,EXOC7,AL135818.2,SEC23A,MT-ND 5,DI 1114P,AC009093.7,CASC1,NCF1,KMT2B,AC073073.1,METTL7A,MRPL57,AL353763.2,AC007298.2,SCML4,AC123768.2,AC136475.7,MIR7847,AC DTROCK1P1 SAFB PBX2 AC020658.7AC018695.6.PSMD10AC105265.4AC018682 1.COLGALT2 RPL39P18,GIMAP5,ZNF83,UQCRH AC073840 AS1,RF00273,RPP30,CYP4F25P,CGAS,SYN1,RNU4-85P,SPHK1,SSSCA1-AS1,ZNF480,SDAD1P1,AP005131.2,AL353763.1,FAM86DP,RF01169,ACA 731P,C10orf143,BLOC1S3,ZSWIM7,ZNF155,AC010530.1,AC026634.1,RBM8A,STYX,PIGO,COX6A1P2,APTX,ATP5MPL,BNIP1,ZNF512B,ODC1-DT,I 45,AC090615.1,AC005726.3,AC007041.1,AC006205.2,SMC04,MANEAL,AC138150.2,LINC00659,RPL39,AC010285.3,CHI3L1,AL109804.1,ARL4C, 553P,PDE4B,AL445187.1,AL136441.1,PDK4,AC109454.2,RF01233,MORN2,TNFAIP8L1,AP003108.4,MIR4769,UQCR10,RAB3A,RNVU1-15,AC0062 14P,AC027559.1,AC080023.2,INSR,AL049780.1,AC124798.1,AC024475.1,RFC3,RPL21P28,AC021422.1,PHLDB2,AC144530.1,HLA-H,MIR6075,WI AS1,AC146507.2,AL031005.1,LINGO2,PDGFA,SIRT7,LTN1,AC022868.1,CNTNAP1,MIR4258,MAP9,WWC2,AL513475.1,FAM189B,AC009090.1,RCS 1143P,MIR3671,SECISBP2,ATP5PD,AC020913.3,FLNB,YIPF5,GPSM2,ZDHHC13,AC023389.1,HLF,AC120114.3,NANP,AC139495.1,AC087501.4,AC 
Table4

The collection of significantly enricher GO pathways (top 10 according to $P$ value) in group CR

\begin{tabular}{|c|c|c|c|c|c|}
\hline ONTOLOGY & ID & Description & Count & $\begin{array}{l}\mathrm{P} \text { - } \\
\text { value }\end{array}$ & GenelD \\
\hline BP & GO:0042119 & $\begin{array}{l}\text { Neutrophil } \\
\text { activation }\end{array}$ & 200 & $\begin{array}{l}1.10 \mathrm{E}- \\
13\end{array}$ & SLC27A2/SLPI/PFKL/PTPRN2/P2RX1/APAF1/LAMP1/ACAA1/FGL2/FCER1G/STXBP3/ \\
\hline BP & GO:0002446 & $\begin{array}{l}\text { Neutrophil } \\
\text { mediated } \\
\text { immunity }\end{array}$ & 199 & $\begin{array}{l}2.81 \mathrm{E}- \\
13\end{array}$ & SLC27A2/SLPI/PFKL/PTPRN2/P2RX1/APAF1/LAMP1/ACAA1/FGL2/FCER1G/STXBP3/ \\
\hline BP & GO:0002283 & $\begin{array}{l}\text { Neutrophil } \\
\text { activation } \\
\text { involved in } \\
\text { immune } \\
\text { response }\end{array}$ & 194 & $\begin{array}{l}7.92 \mathrm{E}- \\
13\end{array}$ & SLC27A2/SLPI/PFKL/PTPRN2/P2RX1/APAF1/LAMP1/ACAA1/FGL2/FCER1G/STXBP3/ \\
\hline BP & G0:0043312 & $\begin{array}{l}\text { Neutrophil } \\
\text { degranulation }\end{array}$ & 193 & $\begin{array}{l}8.15 \mathrm{E}- \\
13\end{array}$ & SLC27A2/SLPI/PFKL/PTPRN2/P2RX1/APAF1/LAMP1/ACAA1/FGL2/FCER1G/STXBP3/ \\
\hline BP & GO:0032543 & $\begin{array}{l}\text { Mitochondrial } \\
\text { translation }\end{array}$ & 70 & $\begin{array}{l}3.01 \mathrm{E}- \\
11\end{array}$ & UQCC2/NSUN3/GATC/COA3/GADD45GIP1/TRMT10C/MTIF3/RMND1/MRPL37/GATB/N \\
\hline $\mathrm{CC}$ & GO:0005743 & $\begin{array}{l}\text { Mitochondrial } \\
\text { inner } \\
\text { membrane }\end{array}$ & 193 & $\begin{array}{l}1.72 \mathrm{E}- \\
14\end{array}$ & TIMM22/SLC25A3/CRLS1/GPD2/UQCC2/COA3/TIMM23/ALAS2/CLU/AP00/SLC25A15 \\
\hline $\mathrm{CC}$ & GO:0098798 & $\begin{array}{l}\text { Mitochondrial } \\
\text { protein } \\
\text { complex }\end{array}$ & 119 & $1.58 \mathrm{E}-$ & TIMM22/NSUN3/COA3/TIMM23/TOMM40L/AP00/TRMT10C/ATP5F1B/PPIF/TIMM21, \\
\hline $\mathrm{CC}$ & GO:0005759 & $\begin{array}{l}\text { Mitochondrial } \\
\text { matrix }\end{array}$ & 179 & $\begin{array}{l}1.27 \mathrm{E}- \\
10\end{array}$ & UQCC2/NSUN3/PCCB/ALAS2/GADD45GIP1/MIPEP/TRMT10C/ATP5F1B/ABHD10/SLC2 \\
\hline $\mathrm{CC}$ & G0:0010008 & $\begin{array}{l}\text { Endosome } \\
\text { membrane }\end{array}$ & 179 & $\begin{array}{l}8.87 \mathrm{E}- \\
10\end{array}$ & SORT1/LAMP1/RABEPK/BAIAP3/CHMP5/GGA3/VPS52/RAB27B/TMEM150B/SNX6/IN \\
\hline $\mathrm{CC}$ & G0:0000315 & $\begin{array}{l}\text { Organellar } \\
\text { large } \\
\text { ribosomal } \\
\text { subunit }\end{array}$ & 36 & $\begin{array}{l}1.25 \mathrm{E}- \\
09\end{array}$ & NSUN3/MRPL37/MRPL32/MRPL51/MRPL42/MRPL43/MPV17L2/MRPL14/MRPL44/M \\
\hline MF & GO:0003713 & $\begin{array}{l}\text { Transcription } \\
\text { coactivator } \\
\text { activity }\end{array}$ & 123 & $\begin{array}{l}1.04 \mathrm{E}- \\
06\end{array}$ & JADE1/PKN1/ASXL1/TAF12/TRERF1/ELF3/TFDP1/TRIP4/MED13/NFYC/FOXC1/ENY2. \\
\hline MF & G0:0140030 & $\begin{array}{l}\text { Modification- } \\
\text { dependent } \\
\text { protein } \\
\text { binding }\end{array}$ & 62 & $\begin{array}{l}9.99 \mathrm{E}- \\
06\end{array}$ & SHARPIN/TP53BP1/MSH6/RNF168/ZMYND11/TNFAIP3/PSME4/SPIN2B/EPS15/L3ME \\
\hline MF & GO:0032182 & $\begin{array}{l}\text { Ubiquitin-like } \\
\text { protein } \\
\text { binding }\end{array}$ & 45 & $\begin{array}{l}1.21 \mathrm{E}- \\
05\end{array}$ & SHARPIN/RNF185/GGA3/DCUN1D5/UCHL3/RNF168/TNFAIP3/SERBP1/DCUN1D3/ASC \\
\hline MF & G0:0003725 & $\begin{array}{l}\text { Double- } \\
\text { stranded RNA } \\
\text { binding }\end{array}$ & 37 & $\begin{array}{l}1.70 \mathrm{E}- \\
05\end{array}$ & AG03/DHX36/RC3H1/ZFR/DROSHA/TFRC/DHX9/MRPL44/FXR1/LSM14A/DDX1/LRRF \\
\hline MF & GO:0000287 & $\begin{array}{l}\text { Magnesium } \\
\text { ion binding }\end{array}$ & 84 & $\begin{array}{l}1.92 \mathrm{E}- \\
05\end{array}$ & IRAK3/ERN1/MSH6/TREX2/DHX36/PPA1/PGM2/ENO2/PIF1/PRPS1/RP2/ARF1/XYLT2. \\
\hline
\end{tabular}

Table5

The collection of significantly enricher GO pathways (top 10 according to P value) in group HR

\section{Table6}

Ten most significantly enriched KEGG pathways in group CR. 


\begin{tabular}{|c|c|c|c|c|c|}
\hline ONTOLOGY & ID & Description & Count & $\begin{array}{l}\mathrm{P}- \\
\text { value }\end{array}$ & GenelD \\
\hline BP & G0:0140053 & $\begin{array}{l}\text { mitochondrial } \\
\text { gene expression }\end{array}$ & 88 & $\begin{array}{l}6.01 \mathrm{E}- \\
15\end{array}$ & NGRN/MRRF/MRPL57/MTG1/TEFM/MRPS2/GATC/MTIF3/TRMT10C/TRNT1/NSUI \\
\hline BP & GO:0042119 & $\begin{array}{l}\text { neutrophil } \\
\text { activation }\end{array}$ & 204 & $\begin{array}{l}2.76 \mathrm{E}- \\
14\end{array}$ & MMP9/ALDOC/MCEMP1/PSMB1/GLIPR1/ERP44/B4GALT1/PSMD2/ORMDL3/LRG1 \\
\hline BP & G0:0002446 & $\begin{array}{l}\text { neutrophil } \\
\text { mediated } \\
\text { immunity }\end{array}$ & 203 & $\begin{array}{l}7.21 \mathrm{E}- \\
14\end{array}$ & MMP9/ALDOC/MCEMP1/PSMB1/GLIPR1/ERP44/B4GALT1/PSMD2/ORMDL3/LRG1 \\
\hline BP & G0:0002283 & $\begin{array}{l}\text { neutrophil } \\
\text { activation } \\
\text { involved in } \\
\text { immune } \\
\text { response }\end{array}$ & 199 & $\begin{array}{l}9.70 \mathrm{E}- \\
14\end{array}$ & MMP9/ALDOC/MCEMP1/PSMB1/GLIPR1/ERP44/B4GALT1/PSMD2/ORMDL3/LRG1 \\
\hline BP & G0:0043312 & $\begin{array}{l}\text { neutrophil } \\
\text { degranulation }\end{array}$ & 198 & $\begin{array}{l}9.84 \mathrm{E}- \\
14\end{array}$ & MMP9/ALDOC/MCEMP1/PSMB1/GLIPR1/ERP44/B4GALT1/PSMD2/ORMDL3/LRG1 \\
\hline $\mathrm{CC}$ & G0:0005743 & $\begin{array}{l}\text { mitochondrial } \\
\text { inner membrane }\end{array}$ & 199 & $\begin{array}{l}2.89 \mathrm{E}- \\
16\end{array}$ & ATP5PO/ATP5MD/RDH13/SLC25A26/PPIF/UQCR10/MCUB/MRPL57/CYC1/AFG3L2 \\
\hline $\mathrm{CC}$ & G0:0098798 & $\begin{array}{l}\text { mitochondrial } \\
\text { protein complex }\end{array}$ & 124 & $\begin{array}{l}1.65 \mathrm{E}- \\
15\end{array}$ & ATP5PO/ATP5MD/PPIF/UQCR10/MCUB/MRPL57/CYC1/AFG3L2/MRPS2/MTX3/TR \\
\hline $\mathrm{CC}$ & GO:0005759 & $\begin{array}{l}\text { mitochondrial } \\
\text { matrix }\end{array}$ & 190 & $\begin{array}{l}1.07 \mathrm{E}- \\
13\end{array}$ & HSPE1/PYCR2/LIAS/PYCR1/ALKBH7/NR3C1/PPIF/STYXL1/TK2/PARS2/MRRF/ECH \\
\hline $\mathrm{CC}$ & G0:0016607 & nuclear speck & 161 & $\begin{array}{l}7.41 \mathrm{E}- \\
12\end{array}$ & SLU7/SRSF8/SLC2A4RG/FBXL4/NR3C1/SMU1/OIP5/ALKBH5/CASC3/POLI/PRPF6/ \\
\hline $\mathrm{CC}$ & GO:0005819 & spindle & 141 & $\begin{array}{l}1.25 \mathrm{E}- \\
10\end{array}$ & MYH9/TBL1X/PLK2/KLHL22/NR3C1/PPP2CA/PAFAH1B1/MAP10/DID01/MZT2A/P \\
\hline MF & G0:0043021 & $\begin{array}{l}\text { ribonucleoprotein } \\
\text { complex binding }\end{array}$ & 63 & $\begin{array}{l}2.43 \mathrm{E}- \\
07\end{array}$ & NAA10/RBM3/SECISBP2/CPEB4/OLA1/EZH2/LTN1/MRRF/NMD3/PRPF6/DDX3X/S \\
\hline MF & GO:0004674 & $\begin{array}{l}\text { protein } \\
\text { serine/threonine } \\
\text { kinase activity }\end{array}$ & 160 & $\begin{array}{l}3.58 \mathrm{E}- \\
06\end{array}$ & CDK7/TGFBR1/PLK2/ERN1/GRK3/CSNK1G3/MAP3K21/ULK4/MAST4/PAK1/VRK3/ \\
\hline MF & GO:0031072 & $\begin{array}{l}\text { heat shock } \\
\text { protein binding }\end{array}$ & 54 & $\begin{array}{l}8.52 \mathrm{E}- \\
06\end{array}$ & ERN1/USP19/NR3C1/DNAJB2/DNAJA2/HSP90AB1/CDKN1B/PPP5C/TELO2/SPN/Z \\
\hline MF & GO:0004842 & $\begin{array}{l}\text { ubiquitin-protein } \\
\text { transferase } \\
\text { activity }\end{array}$ & 139 & $\begin{array}{l}1.67 \mathrm{E}- \\
05\end{array}$ & MALT1/FBXL4/RNF185/WDSUB1/LTN1/FBX040/HERC5/UBE2G2/MIB2/MKRN1/PP \\
\hline MF & G0:0003743 & $\begin{array}{l}\text { translation } \\
\text { initiation factor } \\
\text { activity }\end{array}$ & 28 & $\begin{array}{l}1.82 \mathrm{E}- \\
05\end{array}$ & EIF4G2/MTIF3/EIF4A2/EIF4H/EIF4A1/EIF6/EIF3K/EIF3H/EIF3J/EIF3F/MTIF2/EIF4G: \\
\hline
\end{tabular}

Ten most significantly enriched KEGG pathways in group HR.

\section{Figures}




\begin{tabular}{|c|c|c|c|c|c|c|}
\hline ONTOLOGY & ID & \multicolumn{2}{|l|}{ Description } & Count & $\begin{array}{l}P \text { - } \\
\text { value }\end{array}$ & GenelD \\
\hline BP & G0:0140053 & \multicolumn{2}{|c|}{$\begin{array}{l}\text { mitochondrial } \\
\text { gene expression }\end{array}$} & 88 & $\begin{array}{l}6.01 \mathrm{E}- \\
15\end{array}$ & NGRN/MRRF/MRPL57/MTG1/TEFM/MRPS2/GATC/MTIF3/TRMT10C/TRNT1/NSUN \\
\hline BP & G0:0042119 & \multicolumn{2}{|l|}{$\begin{array}{l}\text { neutrophil } \\
\text { activation }\end{array}$} & 204 & $\begin{array}{l}2.76 \mathrm{E}- \\
14\end{array}$ & MMP9/ALDOC/MCEMP1/PSMB1/GLIPR1/ERP44/B4GALT1/PSMD2/ORMDL3/LRG1 \\
\hline BP & GO:0002446 & \multicolumn{2}{|l|}{$\begin{array}{l}\text { neutrophil } \\
\text { mediated } \\
\text { immunity }\end{array}$} & 203 & $\begin{array}{l}7.21 \mathrm{E}- \\
14\end{array}$ & MMP9/ALDOC/MCEMP1/PSMB1/GLIPR1/ERP44/B4GALT1/PSMD2/ORMDL3/LRG1 \\
\hline BP & GO:0002283 & \multicolumn{2}{|l|}{$\begin{array}{l}\text { neutrophil } \\
\text { activation } \\
\text { involved in } \\
\text { immune } \\
\text { response }\end{array}$} & 199 & $\begin{array}{l}9.70 \mathrm{E}- \\
14\end{array}$ & MMP9/ALDOC/MCEMP1/PSMB1/GLIPR1/ERP44/B4GALT1/PSMD2/ORMDL3/LRG1 \\
\hline BP & G0:0043312 & \multicolumn{2}{|c|}{$\begin{array}{l}\text { neutrophil } \\
\text { degranulation }\end{array}$} & 198 & $\begin{array}{l}9.84 \mathrm{E}- \\
14\end{array}$ & MMP9/ALDOC/MCEMP1/PSMB1/GLIPR1/ERP44/B4GALT1/PSMD2/ORMDL3/LRG1 \\
\hline $\mathrm{CC}$ & G0:0005743 & \multicolumn{2}{|c|}{$\begin{array}{l}\text { mitochondrial } \\
\text { inner membrane }\end{array}$} & 199 & $\begin{array}{l}2.89 \mathrm{E}- \\
16\end{array}$ & ATP5P0/ATP5MD/RDH13/SLC25A26/PPIF/UQCR10/MCUB/MRPL57/CYC1/AFG3L2 \\
\hline $\mathrm{CC}$ & GO:0098798 & \multicolumn{2}{|c|}{$\begin{array}{l}\text { mitochondrial } \\
\text { protein complex }\end{array}$} & 124 & $\begin{array}{l}1.65 \mathrm{E}- \\
15\end{array}$ & ATP5P0/ATP5MD/PPIF/UQCR10/MCUB/MRPL57/CYC1/AFG3L2/MRPS2/MTX3/TR \\
\hline $\mathrm{CC}$ & GO:0005759 & \multicolumn{2}{|c|}{$\begin{array}{l}\text { mitochondrial } \\
\text { matrix }\end{array}$} & 190 & $\begin{array}{l}1.07 \mathrm{E}- \\
13\end{array}$ & HSPE1/PYCR2/LIAS/PYCR1/ALKBH7/NR3C1/PPIF/STYXL1/TK2/PARS2/MRRF/ECF \\
\hline $\mathrm{CC}$ & GO:0016607 & \multicolumn{2}{|c|}{ nuclear speck } & 161 & $\begin{array}{l}7.41 \mathrm{E}- \\
12\end{array}$ & SLU7/SRSF8/SLC2A4RG/FBXL4/NR3C1/SMU1/OIP5/ALKBH5/CASC3/POLI/PRPF6/ \\
\hline $\mathrm{CC}$ & G0:0005819 & \multicolumn{2}{|l|}{ spindle } & 141 & $\begin{array}{l}1.25 \mathrm{E}- \\
10\end{array}$ & MYH9/TBL1X/PLK2/KLHL22/NR3C1/PPP2CA/PAFAH1B1/MAP10/DID01/MZT2A/P \\
\hline MF & G0:0043021 & \multicolumn{2}{|c|}{$\begin{array}{l}\text { ribonucleoprotein } \\
\text { complex binding }\end{array}$} & 63 & $\begin{array}{l}2.43 \mathrm{E}- \\
07\end{array}$ & NAA10/RBM3/SECISBP2/CPEB4/OLA1/EZH2/LTN1/MRRF/NMD3/PRPF6/DDX3X/S \\
\hline MF & GO:0004674 & \multicolumn{2}{|c|}{$\begin{array}{l}\text { protein } \\
\text { serine/threonine } \\
\text { kinase activity }\end{array}$} & 160 & $\begin{array}{l}3.58 \mathrm{E}- \\
06\end{array}$ & CDK7/TGFBR1/PLK2/ERN1/GRK3/CSNK1G3/MAP3K21/ULK4/MAST4/PAK1/VRK3/ \\
\hline MF & GO:0031072 & \multicolumn{2}{|c|}{$\begin{array}{l}\text { heat shock } \\
\text { protein binding }\end{array}$} & 54 & $\begin{array}{l}8.52 \mathrm{E}- \\
06\end{array}$ & ERN1/USP19/NR3C1/DNAJB2/DNAJA2/HSP90AB1/CDKN1B/PPP5C/TELO2/SPN/Z \\
\hline MF & GO:0004842 & \multicolumn{2}{|c|}{$\begin{array}{l}\text { ubiquitin-protein } \\
\text { transferase } \\
\text { activity }\end{array}$} & 139 & $\begin{array}{l}1.67 \mathrm{E}- \\
05\end{array}$ & MALT1/FBXL4/RNF185/WDSUB1/LTN1/FBX040/HERC5/UBE2G2/MIB2/MKRN1/PP \\
\hline MF & GO:0003743 & \multicolumn{2}{|c|}{$\begin{array}{l}\text { translation } \\
\text { initiation factor } \\
\text { activity }\end{array}$} & 28 & $\begin{array}{l}1.82 \mathrm{E}- \\
05\end{array}$ & EIF4G2/MTIF3/EIF4A2/EIF4H/EIF4A1/EIF6/EIF3K/EIF3H/EIF3J/EIF3F/MTIF2/EIF4G: \\
\hline ID & Descripcion & Count & $\begin{array}{l}\mathrm{P} \text { - } \\
\text { value }\end{array}$ & \multicolumn{2}{|c|}{ P.adjust } & GenelD \\
\hline hsa05020 & Prion disease & 116 & $\begin{array}{l}2.04 \mathrm{E}- \\
08\end{array}$ & \multicolumn{2}{|c|}{$6.68 \mathrm{E}-06$} & $=-14.8136129 /-13.83319472 /-41.2285027 /-10.55094562 /-17.72900004 /-2.5623004$ \\
\hline hsa05016 & $\begin{array}{l}\text { Huntington } \\
\text { disease }\end{array}$ & 126 & $\begin{array}{l}4.64 \mathrm{E}- \\
08\end{array}$ & \multicolumn{2}{|c|}{ 7.61E-06 } & $=-13.83319472 /-41.2285027 /-10.55094562 /-24.48111328 /-173.0376027 /-17.72900$ \\
\hline hsa05012 & Parkinson disease & 103 & $\begin{array}{l}6.15 \mathrm{E}- \\
07\end{array}$ & \multicolumn{2}{|c|}{$5.08 \mathrm{E}-05$} & $=-13.83319472 /-41.2285027 /-10.55094562 /-24.48111328 /-17.72900004 /-2.562300$ \\
\hline hsa04144 & Endocytosis & 104 & $\begin{array}{l}6.20 \mathrm{E}- \\
07\end{array}$ & \multicolumn{2}{|c|}{ 5.08E-05 } & $=-9.971260833 /-80.55898765 /-10.43336139 /-4.052067933 /-32.50125904 /-4.06412$ \\
\hline hsa05010 & Alzheimer disease & 142 & $\begin{array}{l}8.51 \mathrm{E}- \\
07\end{array}$ & \multicolumn{2}{|c|}{$5.58 \mathrm{E}-05$} & $=-13.83319472 /-41.2285027 /-10.55094562 /-24.48111328 /-173.0376027 /-17.72900$ \\
\hline hsa03050 & Proteasome & 28 & $\begin{array}{l}1.60 \mathrm{E}- \\
06\end{array}$ & \multicolumn{2}{|c|}{ 8.75E-05 } & $=-25.54134178 /-41.2285027 /-10.55094562 /-17.72900004 /-11.60909027 /-5.543828$ \\
\hline hsa05014 & $\begin{array}{l}\text { Amyotrophic } \\
\text { lateral sclerosis }\end{array}$ & 137 & $\begin{array}{l}5.37 \mathrm{E}- \\
06\end{array}$ & \multicolumn{2}{|c|}{0.000211928} & $=-13.83319472 /-41.2285027 /-10.55094562 /-24.48111328 /-173.0376027 /-17.72900$ \\
\hline hsa05022 & $\begin{array}{l}\text { Pathways of } \\
\text { neurodegeneration } \\
\text { - multiple diseases }\end{array}$ & 172 & $\begin{array}{l}5.46 \mathrm{E}- \\
06\end{array}$ & \multicolumn{2}{|c|}{0.000211928} & $=-13.83319472 /-41.2285027 /-10.55094562 /-24.48111328 /-173.0376027 /-17.72900$ \\
\hline hsa00190 & $\begin{array}{l}\text { Oxidative } \\
\text { phosphorylation }\end{array}$ & 60 & $\begin{array}{l}5.82 \mathrm{E}- \\
06\end{array}$ & \multicolumn{2}{|c|}{0.000211928} & $=-13.83319472 /-6.454919377 /-32.89604088 /-2.431090716 /-11.55958762 /-33.2431$ \\
\hline hsa04140 & $\begin{array}{l}\text { Autophagy - } \\
\text { animal }\end{array}$ & 61 & $\begin{array}{l}8.16 \mathrm{E}- \\
06\end{array}$ & \multicolumn{2}{|c|}{0.000267488} & $=-17.51223489 /-24.48111328 /-173.0376027 /-6.460668104 /-3.517128856 /-9.05244$ \\
\hline
\end{tabular}




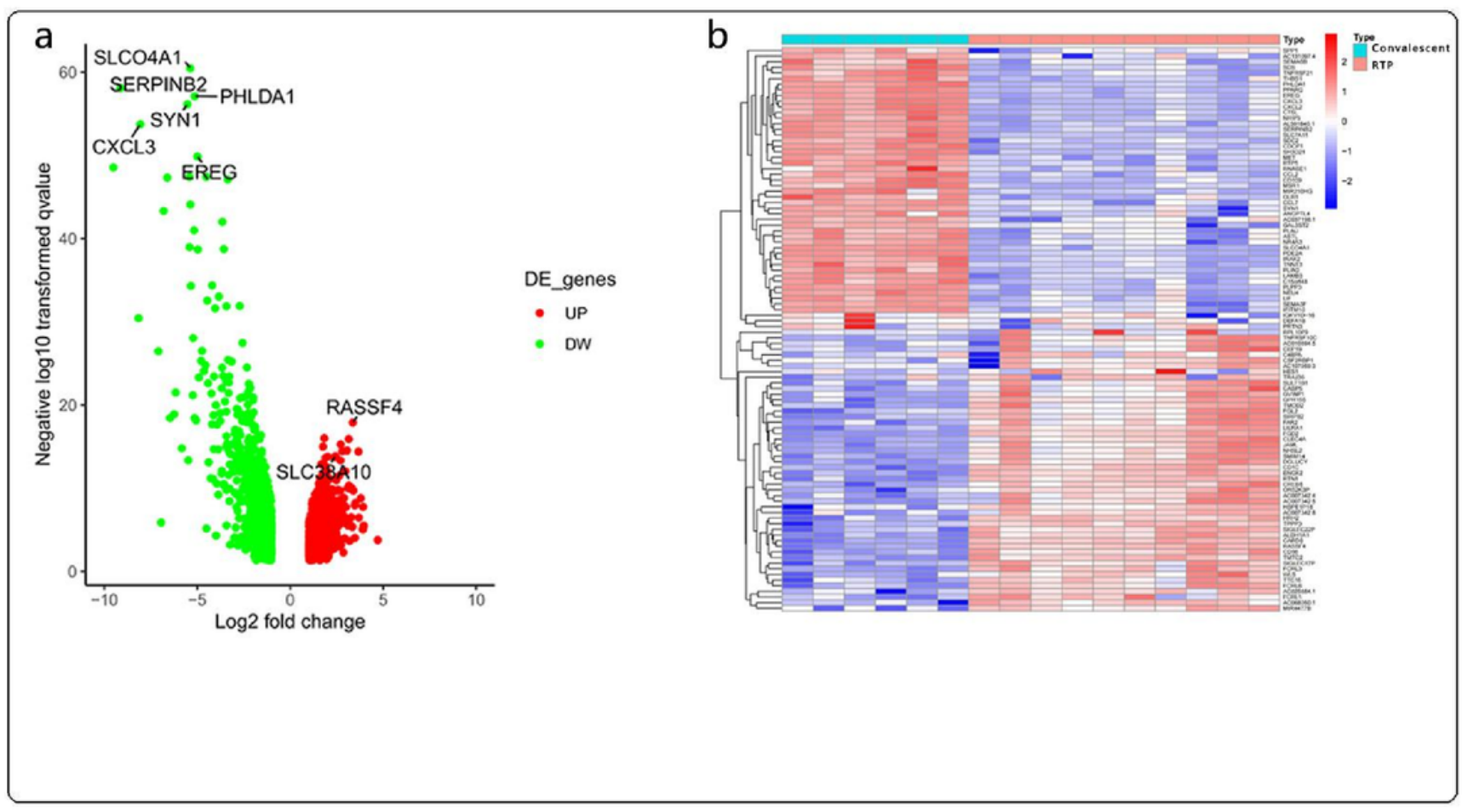

Figure 1

(a) Volcano plot of DEGs in group CR; (b) The heatmap of DEGs in group CR. The colors in (a) indicate different gene expressions in the corresponding group (red for upregulated DEGs and green for downregulated DEGs, gray indicates no difference); for (b), the abscissa axis represents the sample types and the ordinate axis represents the gene names. 


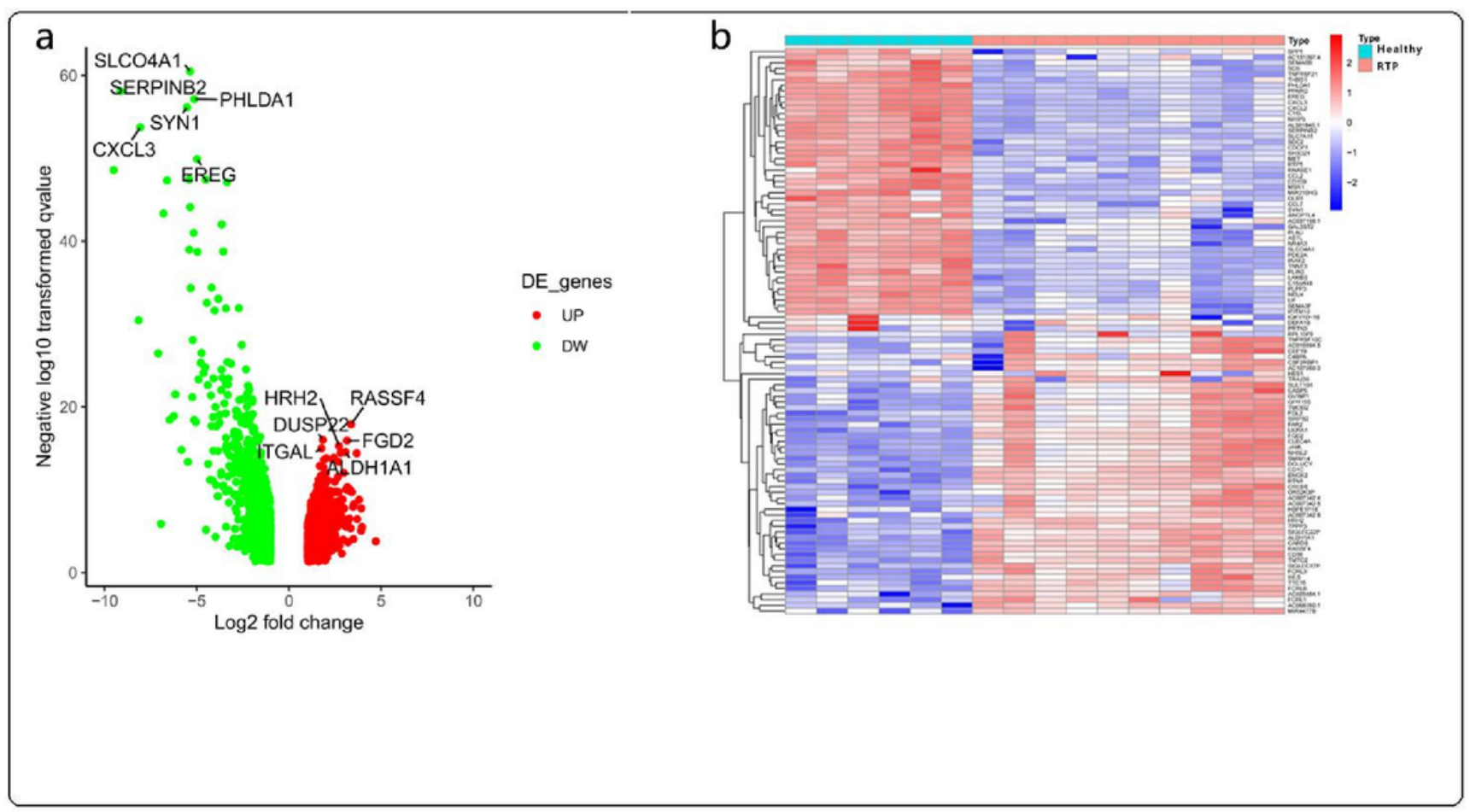

Figure 2

(a)Volcano plot of DEGs in group HR; (b) The heatmap of DEGs in group HR. The colors in (a) indicate different gene expressions in the corresponding group (red for up-regulated DEGs and green for down-regulated DEGs, gray indicates no difference); for (b), the abscissa axis represents the sample types and the ordinate axis represents the gene names. 


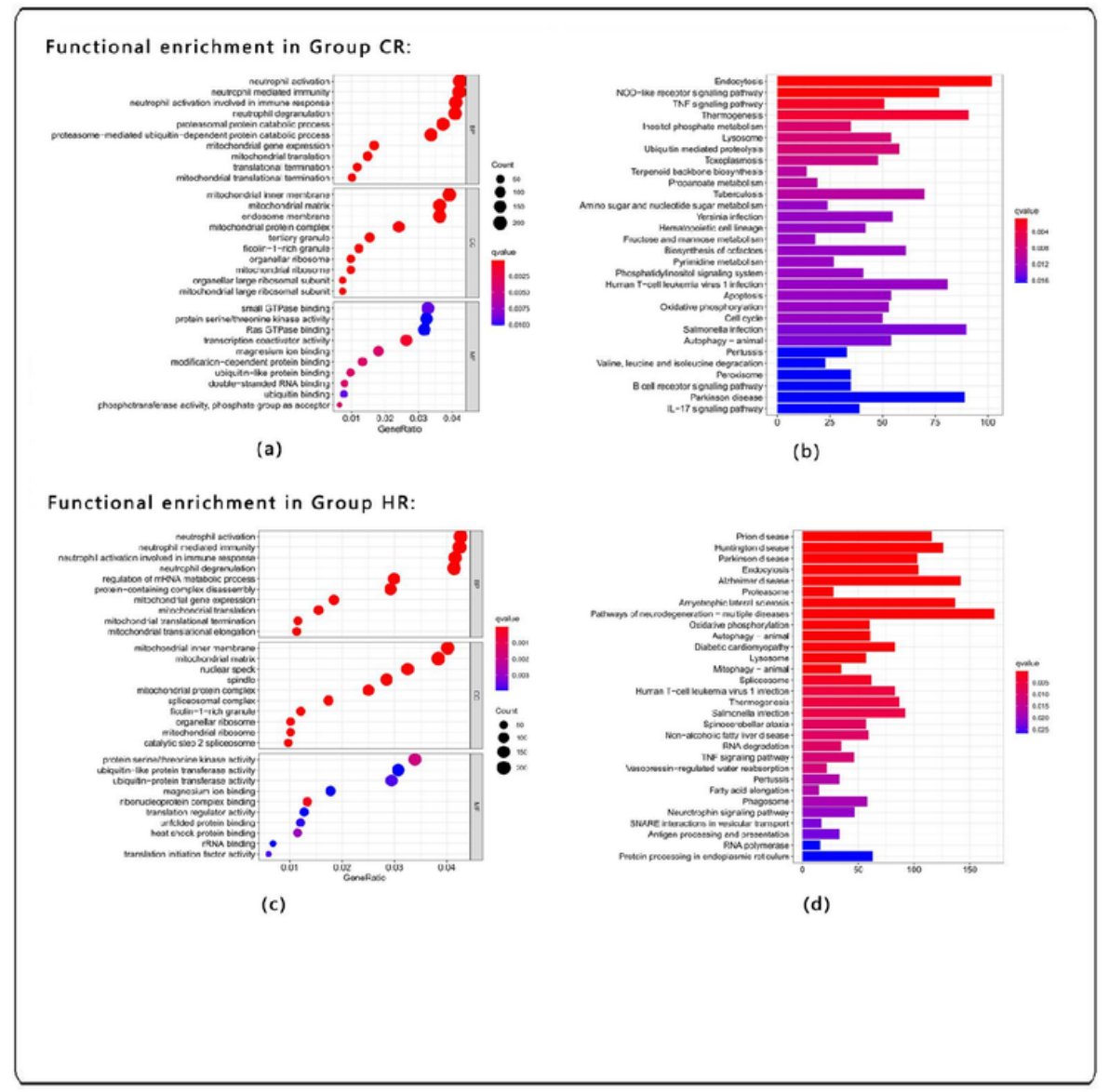

\section{Figure 3}

Results of functional analysis in group CR and group HR (a) The GO analysis of group CR; (b) The KEGG analysis of group CR; (c) The GO analysis of group HR; (d) The KEGG analysis of group HR. In (a) and (c), the abscissa axis represents the gene ratio while the ordinate axis represents term names. The size of a single bubble represents the degree of enrichment; the color variety represents the different q-values (those in red are considered to be of significance). For (b) and (d), the abscissa axis represents counts; the ordinate axis represents KEGG pathways; color represents the same meaning as the bubble diagram. 


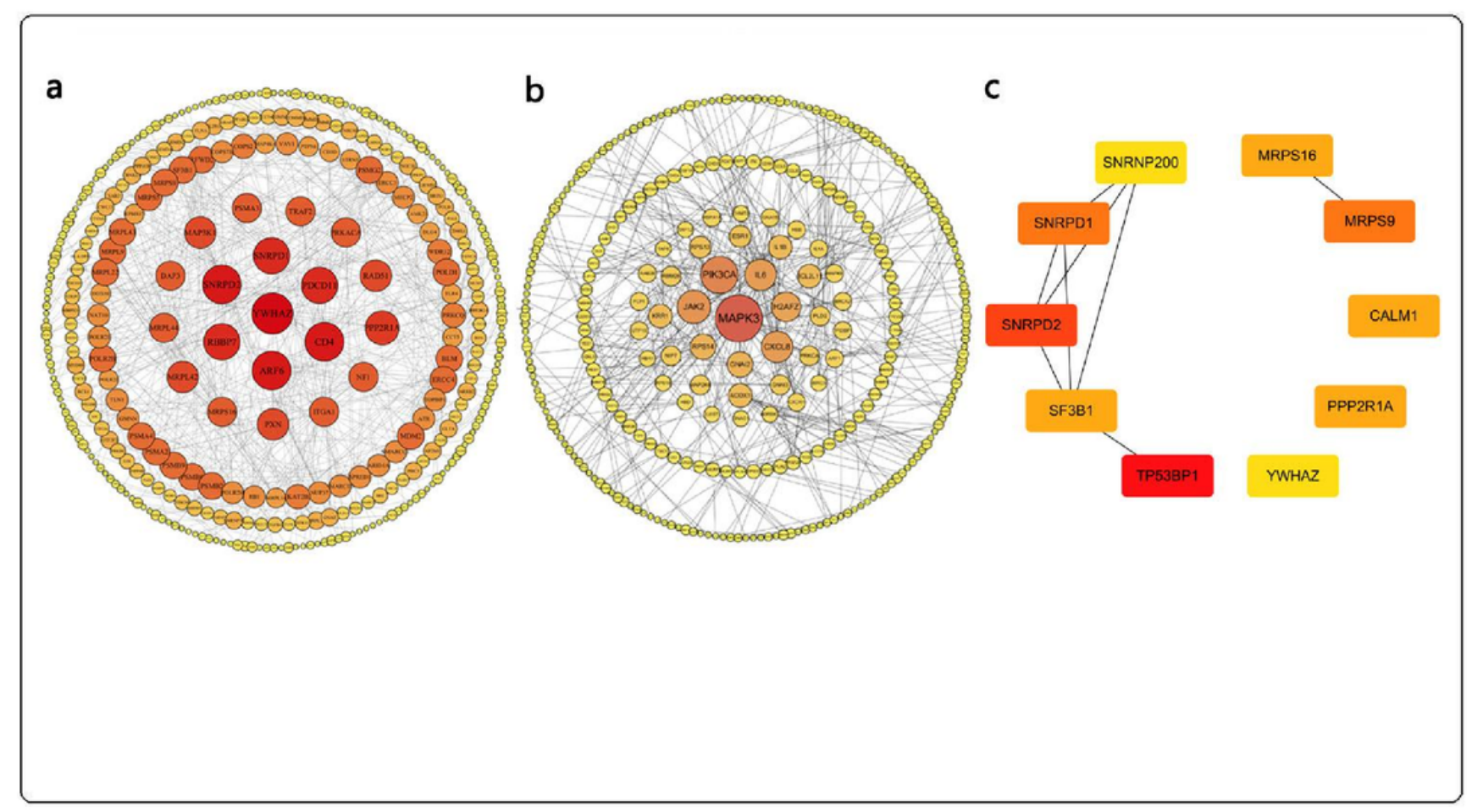

\section{Figure 4}

PPI network of up-regulated genes in group CR; (b)PPI network of down-regulated genes in group CR; (c)Hub genes in group CR. Nodes represent genes; lines represent the interactions between gene-encoded proteins; the redder the color, the more significant it is. 


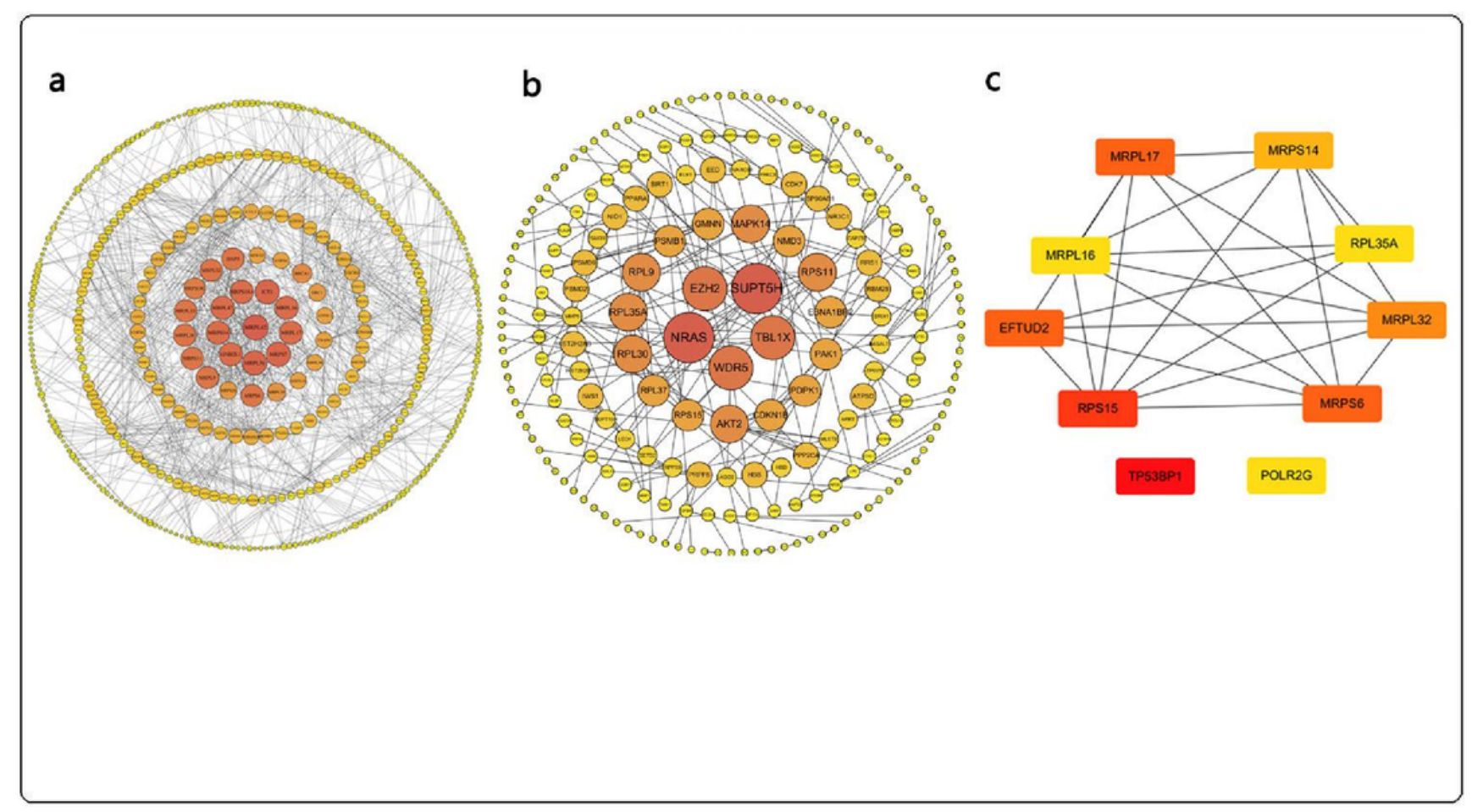

\section{Figure 5}

(a)PPI network of up-regulated genes in group HR; (b)PPI network of down-regulated genes in group HR; (c)Hub genes in group HR. Nodes represent genes; lines represent the interactions between gene-encoded proteins; the redder the color, the more significant it is.

\section{Supplementary Files}

This is a list of supplementary files associated with this preprint. Click to download.

- Additionalfile1.pdf

- Additionalfile2.pdf

- Additionalfile3.pdf 